\title{
Atualização no Tratamento da Hipertensão Arterial Pulmonar
}

\author{
Update on the Treatment of Pulmonary Arterial Hypertension
}

Caio J. Fernandes, ${ }^{1,2,3}$ Daniela Calderaro, ${ }^{1,4}$ Ana Paula Luppino Assad, ${ }^{1,3,5}$ William Salibe-Filho, ${ }^{1}$ Luciana Tamie KatoMorinaga, ${ }^{1,3}$ Susana Hoette, ${ }^{1}$ Bruna Piloto, ${ }^{1,3}$ Marcela Araújo Castro, ${ }^{1,3}{ }^{1,3}$ Roberta Pontes Lisboa, ${ }^{1,3}$ Taysa Antonia Felix da Silva, ${ }^{1,3}$ Murillo de Araújo Martins, ${ }^{1}$ Jose L. Alves-Jr, ${ }^{1,2,3}$ Carlos Jardim, ${ }^{1,3}$ Mario Terra-Filho, ${ }^{1,3}$ Rogerio de Souza ${ }^{1,3}$

Unidade de Circulação Pulmonar - Divisão de Pneumologia - Incor - Faculdade de Medicina da Universidade de São Paulo, ${ }^{1}$ São Paulo, SP - Brasil Instituto do Câncer da Faculdade de Medicina da Universidade de São Paulo, ${ }^{2}$ São Paulo, SP - Brasil

Hospital Sírio-Libanês, ${ }^{3}$ São Paulo, SP - Brasil

Unidade de Medicina Interdisciplinar - Divisão de Cardiologia - Incor - Faculdade de Medicina da Universidade de São Paulo, ${ }^{4}$ São Paulo, SP - Brasil Disciplina de Reumatologia do Hospital das Clínicas da Faculdade de Medicina da Universidade de São Paulo, ${ }^{5}$ São Paulo, SP - Brasil

\section{Resumo}

Muitos avanços ocorreram nas últimas décadas na terapêutica da hipertensão arterial pulmonar (HAP), uma doença grave, progressiva, incurável e potencialmente fatal. Para seu tratamento adequado, são fundamentais o diagnóstico hemodinâmico e a classificação de sua etiologia, em que várias delas (colagenoses, hipertensão portal, cardiopatia congênitas, esquistossomose) requerem medidas específicas, além do tratamento farmacológico característico para HAP.

O tratamento com fármacos-alvo para HAP baseia-se em produtos farmacêuticos que interferem em três vias fisiopatológicas moleculares: da prostaciclina, da endotelina e do óxido nítrico. Tais fármacos apresentam múltiplas apresentações (oral, endovenosa, subcutânea e inalatória) e mudaram a história da HAP. Essas medicações e suas estratégias de uso, assim como particularidades das diferentes formas de HAP, são o foco desta revisão.

\section{Introdução}

A hipertensão arterial pulmonar (HAP) é uma condição clínica que cursa com remodelamento do território vascular pulmonar, levando à obliteração dele e consequente aumento da resistência vascular. ${ }^{1}$ A elevação da resistência acarreta aumento das pressões do sistema, representando o aumento da carga imposta ao ventrículo direito, que evolui com progressiva insuficiência, sendo a principal causa dos sintomas associados à doença. ${ }^{2}$

Trata-se de uma situação clínica rara e grave, acometendo entre 2 a 5 pacientes por milhão de adultos por ano, ${ }^{3} \mathrm{com}$ mediana de sobrevida de 2,8 anos na ausência de tratamento

\section{Palavras-chave}

Hipertensão arterial pulmonar; Hipertensão Pulmonar; Diagnóstico; Terapêutica.

Correspondência: Caio J. Fernandes •

Unidade de Circulação Pulmonar - Divisão de Pneumologia - Incor -

Faculdade de Medicina da Universidade de São Paulo - Av. Dr. Enéas de

Carvalho Aguiar, 44. CEP 05403-000, São Paulo, SP - Brasil

E-mail: cjcfernandes@yahoo.com.br

Artigo recebido em 24/06/2020, revisado em 13/09/2020, aceito em 04/11/2020

DOI: https://doi.org/10.36660/abc.20200702 específico. ${ }^{4}$ Todavia, uma série de medicamentos, com múltiplas apresentações (oral, endovenosa, subcutânea e inalatória) foram desenvolvidos a partir da década de 1990, mudando sobremaneira a realidade da $\mathrm{HP}^{5}$ e seu impacto na qualidade de vida dos pacientes. ${ }^{6}$ Dados do registro francês de HAP demonstraram que após a introdução da terapêutica específica, a sobrevida dos pacientes passou a ser de $82,9 \%$ em 1 ano e de 58,2\% em 3 anos, o que corresponde a uma melhoria estimada em pelo menos $15 \%$ quando comparada com a sobrevida prevista sem o acesso ao tratamento farmacológico. ${ }^{7}$ Tais medicamentos, suas estratégias de uso e as particularidades das diferentes formas de HAP são o foco desta revisão.

\section{Definições}

Define-se hipertensão pulmonar (HP) como uma condição em que a pressão média de artéria pulmonar (PMAP) é superior a $20 \mathrm{mmHg} .{ }^{8}$ Classicamente, utilizou-se o nível de $25 \mathrm{mmHg}$ para defini-la, ${ }^{9}$ mas estudos recentes sugerem que níveis menores já estão associados ao pior prognóstico. ${ }^{10}$ Concomitantemente, se a pressão de oclusão de artéria pulmonar (POAP) for menor ou igual a $15 \mathrm{mmHg}$, temos a condição de HP pré-capilar; nessa situação, o predomínio da doença vascular está no território arterial. Quando a POAP for superior a $15 \mathrm{mmHg}$, temos a condição de HP pós-capilar, o que sugere a presença de alterações nas câmaras cardíacas esquerdas. Baseado nestas definições, representadas na Figura 1, vê-se que o cateterismo cardíaco direito é fundamental para a caracterização adequada da HP. ${ }^{11}$ Vale a pena ressaltar que, enquanto os atuais critérios de HAP incluem valores de resistência vascular pulmonar (RVP) superiores a $3 \mathrm{~W}$, dados recentes indicam que valores superiores a 2,2W apresentam impacto negativo na sobrevida desta população e podem

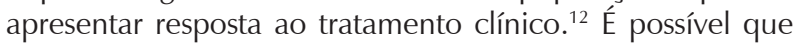
futuras definições de HAP incluam, além do critério pressórico de $20 \mathrm{mmHg}$, valores de RVP superiores a 2,2W.

\section{Classificação}

A partir das definições hemodinâmicas e agregando características fisiopatológicas, clínicas e de tratamento das diferentes etiologias causadoras de HP, é possível classificar os casos em 5 grupos distintos. ${ }^{8,13}$ Considera-se do grupo I os pacientes que tenham doença arterial pulmonar predominante, na ausência de doença pulmonar ou trombembólica, o foco desta revisão. O grupo II inclui pacientes cuja gênese da 


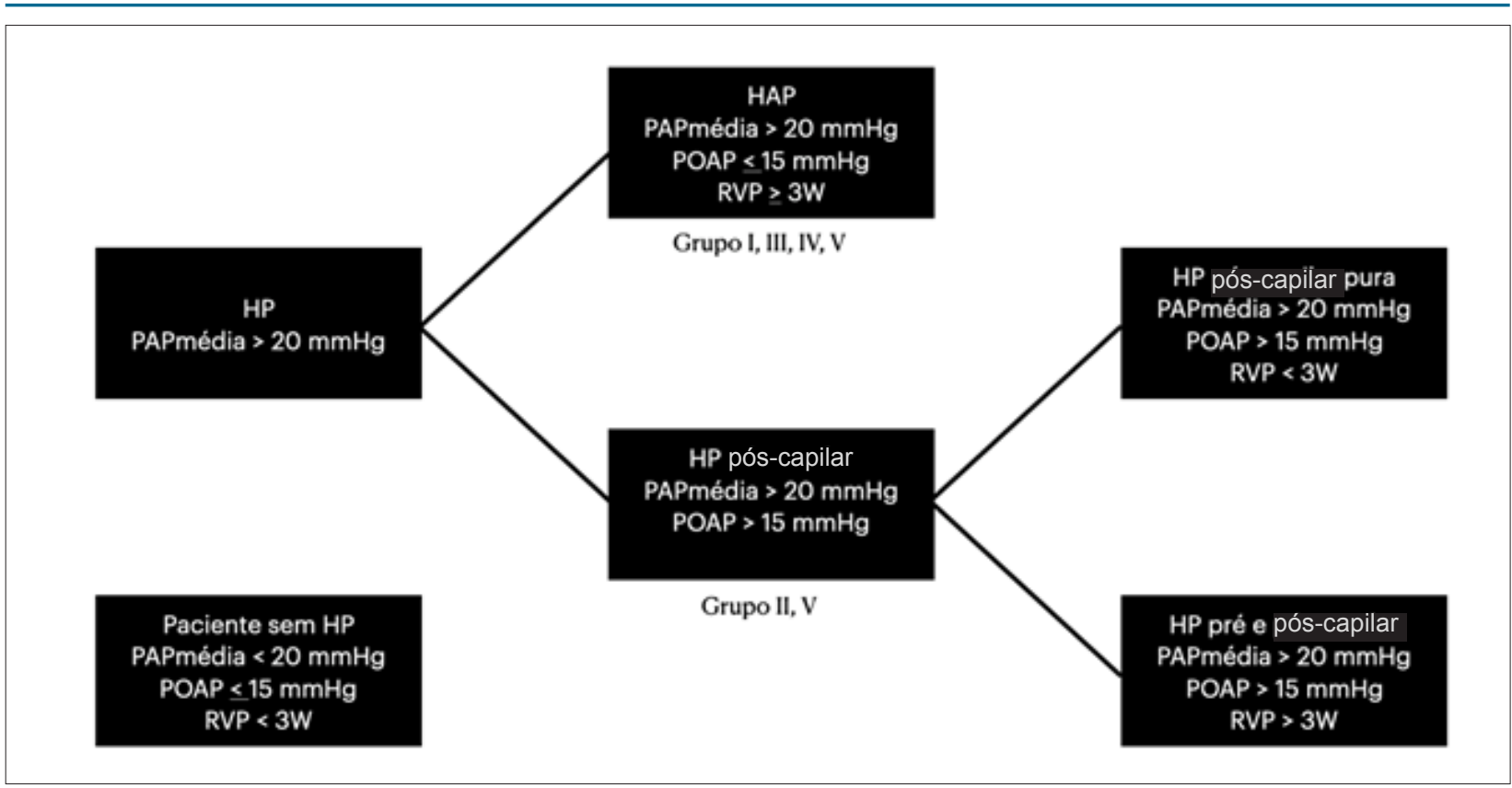

Figura 1 - Definições de alterações hemodinâmicas do sistema vascular pulmonar e sua correlação com os grupos da classificação de hipertensão pulmonar. HP: hipertensão pulmonar; HAP: hipertensão arterial pulmonar; PMAP: pressão média de artéria pulmonar média; POAP: pressão de oclusão de artéria pulmonar; RVP: resistência vascular pulmonar; W: Woods; mmHg: milimetros de mercúrio.

HP seja doença cardíaca esquerda e aumento da pressão hidrostática do sistema, a partir do átrio esquerdo. ${ }^{14} \mathrm{O}$ grupo III inclui pacientes com HP por doença pulmonar crônica, nos quais a gênese da enfermidade advém da perda do leito vascular pulmonar e da vasoconstrição hipóxica. ${ }^{15} \mathrm{O}$ grupo IV inclui pacientes com HP por embolia pulmonar crônica (HPTEC), e possui um manejo clínico distinto, além do escopo deste texto. Recomendações para diagnóstico e tratamento dos pacientes com HPTEC podem ser encontradas em outras publicações. ${ }^{16} \mathrm{O}$ grupo $\mathrm{V}$ inclui pacientes com doenças mais raras, com múltiplos mecanismos. ${ }^{8}$ As diferentes etiologias de HP e sua classificação em grupos estão descritos na Tabela 1. Vale ressaltar que, embora esteja fora do escopo desta revisão, o processo diagnóstico para correta classificação dos casos de HP é bastante extenso e abrangente, garantindo que as estratégias de tratamento sejam adequadas aos mecanismos fisiopatológicos predominantes como causa da elevação pressórica no território vascular pulmonar. ${ }^{17}$

\section{Tratamento da HAP}

\section{Medidas gerais}

Após a confirmação diagnóstica da HAP, medidas gerais que objetivem minimizar as consequências da doença devem ser implementadas. Nesse contexto geral, três delas se destacam: evitar a gestação (condição associada a um agravamento da condição hemodinâmica, pela necessidade de aumento do débito cardíaco durante a gestação, resultando em elevada mortalidade materno-fetal), ${ }^{18}$ realizar imunização para influenza e pneumococo ${ }^{9}$ e o oferecer apoio psicossocial aos portadores de HAP. ${ }^{19}$
Além das que foram citadas, outras medidas que podem ser adotadas são: uso de diuréticos, administração suplementar de oxigênio e a não realização de exercícios físicos extenuantes. Exercícios físicos, com o objetivo de reabilitação, podem ser recomendados, mas sob supervisão, ${ }^{20}$ após o início de tratamento farmacológico específico.

Estudos da década de $1980^{21}$ sugeriam o benefício de anticoagulação, baseando-se nos fenômenos de trombose in situ presentes em estudos de biópsias pulmonares na HAP. No entanto, dados mais recentes ${ }^{22}$ sugerem benefício da anticoagulação apenas para pacientes com hipertensão arterial pulmonar idiopática (HAPI), hereditária ou associada ao uso de anorexígenos. Dessa forma, a indicação de anticoagulação em HAP deixou de ser uma conduta universal e passou a estar baseada, caso a caso, na avaliação de risco/benefício. ${ }^{23}$

\section{Avaliação da vasorreatividade}

Para pacientes com HAPI, hereditária ou induzida por fármacos, o teste de vasorreatividade deve ser realizado durante o cateterismo cardíaco direito diagnóstico. O padrão-ouro utiliza a inalação de óxido nítrico 10 a 20 ppm durante 10 minutos. O teste é positivo quando há uma queda maior de $10 \mathrm{mmHg}$ na PMAP, alcançando valores abaixo de $40 \mathrm{mmHg}$ e sem redução do débito cardíaco (DC). ${ }^{2}$ O teste de vasorreatividade visa a identificar um subgrupo de pacientes nos quais o aumento do tônus vascular seja o mecanismo predominante na gênese da HAP, não o remodelamento vascular. ${ }^{24}$

Pacientes com HAP que apresentem resposta positiva ao teste de vasorreatividade devem ser submetidos ao tratamento com bloqueador de canal de cálcio (BCC), 


\section{Artigo de Revisão}

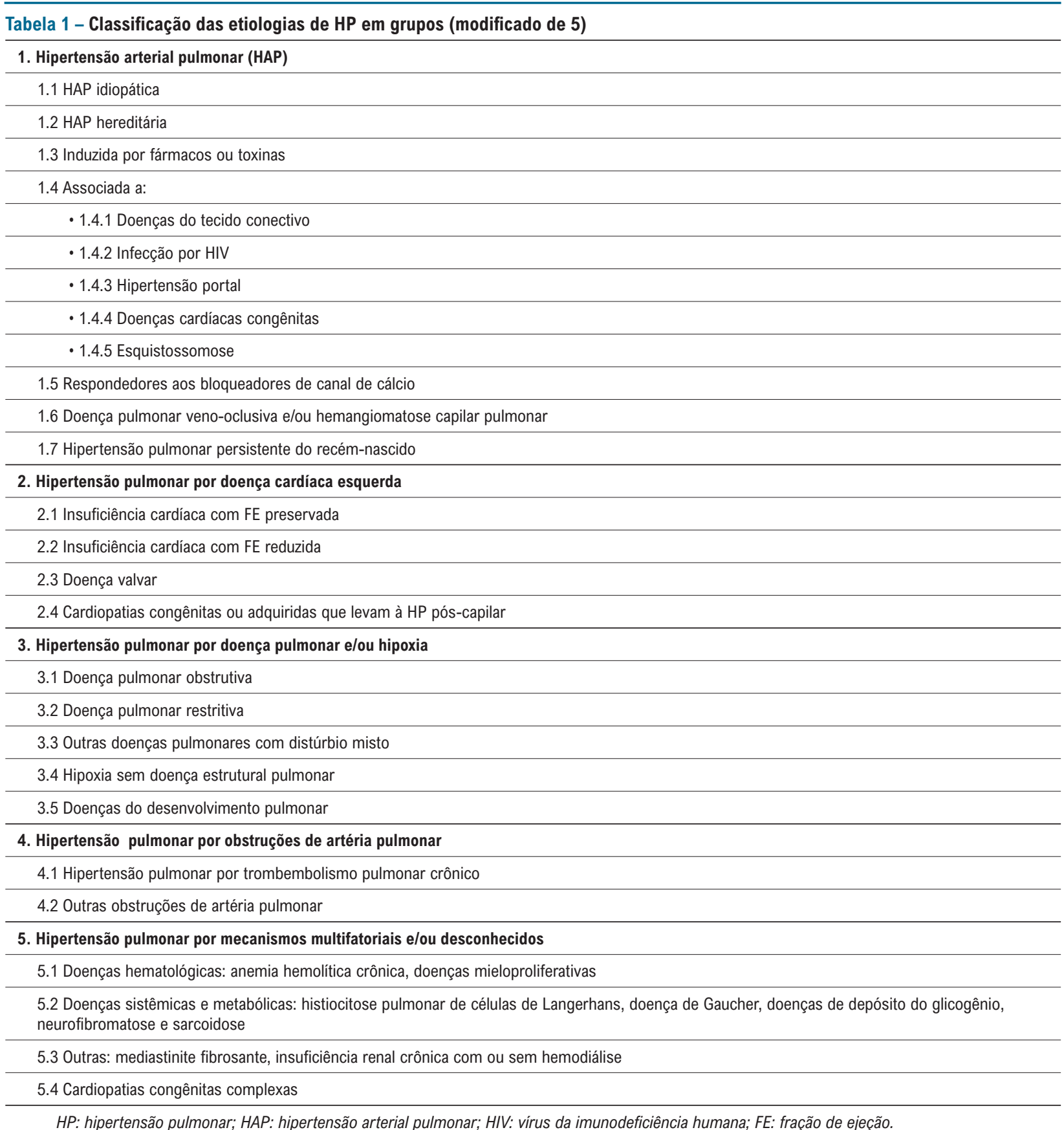

HP: hipertensão pulmonar; HAP: hipertensão arterial pulmonar; HIV: vírus da imunodeficiência humana; FE: fração de ejeção.

preferencialmente de ação prolongada e com a maior dose tolerada. ${ }^{25}$ Sugere-se iniciar com $10 \mathrm{mg}$ de anlodipino $1 \mathrm{x} /$ dia, diltiazem $60 \mathrm{mg} 3 x /$ dia ou nifedipina 30mg $2 x /$ dia. Pacientes que não evoluam para classes funcionais (CF) I/ II e não apresentem melhora hemodinâmica devem receber medicação específica para HAP. Cerca de 12,6\% dos pacientes diagnosticados com HAPI apresentam resposta aguda à vasodilatação; porém, metade não persiste com boa resposta clínica ao uso de BCC após um ano de seguimento. ${ }^{26}$ Pacientes que não tenham feito teste de vasorreatividade não devem ser tratados com BCC. ${ }^{23}$

\section{Tratamento específico}

\section{Vias de tratamento}

O principal achado anatomopatológico na HAP é o remodelamento vascular pulmonar, com espessamento das camadas íntima e camada média. ${ }^{27}$ A vasoconstrição também possui importante papel no desenvolvimento da doença, com aumento do tônus vascular e proliferação das células musculares lisas (CML) das arteríolas. ${ }^{25}$ Três vias fisiopatológicas relacionadas aos mecanismos moleculares que geram esses 
achados histológicos foram identificadas e passaram a ser alvos para o tratamento farmacológico da HAP: da prostaciclina, da endotelina e do óxido nítrico. ${ }^{28}$

\section{Via da prostaciclina}

A prostaciclina $\left(\mathrm{PGI}_{2}\right)$ é uma molécula derivada do ácido araquidônico, capaz de atuar em receptores transmembranas de múltiplos tecidos, com variadas ações biológicas. ${ }^{29} \mathrm{Na}$ circulação pulmonar, ao estimular o receptor IP, a $\mathrm{PGI}_{2}$ é capaz de induzir o relaxamento das CML das arteríolas pulmonares, com consequente vasodilatação, e inibir a sua proliferação, ao estimular a produção do AMP cíclico. ${ }^{30}$ Por outro lado, outra molécula da via da $\mathrm{PGI}_{2}$, o tromboxano $\mathrm{A}_{2}$ $\left(\mathrm{TXA}_{2}\right)$ contrabalança seu efeito, promovendo vasoconstrição e aumento da agregação plaquetária. Na HAP, os níveis de $\mathrm{PGI}_{2}$ e a atividade enzimática da prostaciclina sintase estão significativamente reduzidos, e seu equilíbrio pende em direção ao TXA $2{ }^{31}$ Dessa forma, uma estratégia para o tratamento de HAP é atuar diretamente no receptor IP, com reposição direta da $\mathrm{PGI}_{2}$ (epoprostenol) ${ }^{32}$ ou com fármacos de estrutura análoga à da $\mathrm{PGI}_{2}$ (por exemplo: treprostinil, beraprost e iloprost). ${ }^{33,34}$ Existem, ainda, fármacos capazes de atuar no receptor IP, com estrutura molecular distinta da $\mathrm{PGI}_{2}$ (por exemplo, selexipague). ${ }^{35}$

\section{A via da endotelina}

A endotelina-1 (ET1) é o mais potente vasoconstritor natural de sistemas biológicos, ${ }^{36}$ seus níveis são elevados tanto no endotélio vascular pulmonar quanto no sangue de pacientes com HAP. ${ }^{37}$ A ET1 age por meio de dois tipos de receptores: os receptores de endotelina $A\left(E T_{A}\right)$ e $B\left(E T_{B}\right)$. A bosentana ${ }^{38} \mathrm{e}$ a macitentana ${ }^{39}$ são antagonistas dos receptores de endotelina (ARE), que bloqueiam de forma não seletiva os receptores $\mathrm{A} e$ $\mathrm{B}$, enquanto a ambrisentana ${ }^{40}$ apresenta maior afinidade pelo receptor $\mathrm{ET}_{\mathrm{A}}$. Todas as três substâncias são fármacos orais e mostraram-se eficientes no tratamento da HAP.

\section{A via do óxido nítrico}

O NO é um potente vasodilatador endógeno, que atua nas CML vasculares através do estímulo da guanilil ciclase (GC), levando à produção do GMP cíclico. ${ }^{41}$ Pacientes com HAP apresentam níveis reduzidos de $\mathrm{NO}$ sérico e tecidual. ${ }^{42} \mathrm{~A}$ fosfodiesterase do tipo 5 (PDE-5) é a enzima responsável pela degradação do GMP cíclico. A inibição da PDE-5 leva a um aumento na concentração de GMP cíclico e ao consequente relaxamento das $\mathrm{CML}$, promovendo vasodilatação. ${ }^{43}$ Sildenafil, tadalafil e vardenafil são os fármacos inibidores da PDE-5 (PDE-5i) disponíveis.

Atuando em outro ponto da via do NO, há o riociguate, um estimulante da GC. Ele potencializa a atividade da GC e a ativa de forma independente ao NO. ${ }^{44}$ A GC estimulada pelo riociguate potencializa a conversão de GTP em GMP cíclico, promovendo vasodilatação. O fármaco pode ser utilizado tanto em HAP ${ }^{45}$ quanto em HPTEC. ${ }^{46}$

Os fármacos disponíveis para o tratamento de HAP no Brasil estão descritos na Tabela 2.

\section{Estratégias de tratamento}

Ao longo dos últimos anos, houve significativa mudança na estratégia de uso dos diferentes fármacos disponíveis, com indicação cada vez mais precoce de tratamento e combinação de fármacos de diferentes vias/classes.

O uso combinado de fármacos apoia-se no potencial de sinergia existente na intervenção simultânea em diferentes vias fisiopatológicas. Comparada com a monoterapia, a associação adicional de um segundo medicamento (terapia combinada sequencial) mostrou-se benéfica para diferentes combinações de fármacos administrados. ${ }^{35,39,47}$ Tais resultados foram reforçados em uma metanálise de 14 estudos de terapia combinada sequencial e que mostrou redução de piora clínica quando comparada com a monoterapia..$^{48}$

Tabela 2 - Fármacos autorizados e disponíveis no Brasil para o tratamento de HAP

\begin{tabular}{|c|c|c|c|c|c|}
\hline Fármacos & Posologia & Via de administração & Efeitos colaterais mais frequentes & Via & Referência \\
\hline lloprost & $\begin{array}{l}2,5-5 \mathrm{mcg} \\
6-9 x \text { ao dia }\end{array}$ & Inalatória & $\begin{array}{c}\text { Tosse } \\
\text { Efeitos irritativos locais }\end{array}$ & $\mathrm{PGI}_{2}$ & 34 \\
\hline Selexipague & $\begin{array}{l}200-1600 \mathrm{mcg} \\
2 x \text { ao dia }\end{array}$ & Oral & $\begin{array}{l}\text { Cefaleia } \\
\text { Diarreia }\end{array}$ & $\mathrm{PGI}_{2}$ & 35 \\
\hline Ambrisentana & $\begin{array}{l}5-10 \mathrm{mg} \\
1 \times \text { ao dia }\end{array}$ & Oral & $\begin{array}{l}\text { Anemia } \\
\text { Edema }\end{array}$ & ET1 & 40 \\
\hline Bosentana & $\begin{array}{l}62,5-125 \mathrm{mg} \\
2 \times \text { ao dia }\end{array}$ & Oral & $\begin{array}{c}\text { Anemia } \\
\text { Hepatotoxicidade }\end{array}$ & ET1 & 38 \\
\hline Macitentana & $\begin{array}{c}10 \mathrm{mg} \\
1 \mathrm{x} \text { ao dia }\end{array}$ & Oral & $\begin{array}{c}\text { Anemia } \\
\text { Hepatotoxicidade } \\
\text { Edema }\end{array}$ & ET1 & 39 \\
\hline Sildenafil & $\begin{array}{l}20-80 \mathrm{mg} \\
3 \mathrm{x} \text { ao dia }\end{array}$ & Oral & Cefaleia & NO & 43 \\
\hline Riociguate & $\begin{array}{c}0,5-2,5 \mathrm{mg} \\
3 x \text { ao dia }\end{array}$ & Oral & $\begin{array}{c}\text { Cefaleia } \\
\text { Hipotensão }\end{array}$ & NO & 45 \\
\hline
\end{tabular}

$P G I_{\text {: }}$ prostaciclina; ET1: endotelina; NO: óxido nítrico. 


\section{Artigo de Revisão}

Em diferente abordagem, um grande estudo clínico avaliou o uso combinado de fármacos desde o diagnóstico, mais precisamente a associação de tadalafil e ambrisentana. ${ }^{47}$ Esta combinação levou a uma redução de $50 \%$ no desfecho combinado de piora clínica quando comparada com a terapia com qualquer um dos medicamentos isoladamente, sem diferença significativa em termos de efeitos colaterais.

Embora não haja estudos comparando diretamente a combinação inicial versus sequencial, a evidência sugere que o tratamento combinado inicial seja bem tolerado e benéfico, mesmo em pacientes considerados de baixo risco. ${ }^{47}$ Dessa forma, a recomendação atual é de que a terapia combinada oral seja considerada ao diagnóstico, conforme detalhado na Figura 2.

No entanto, para uma minoria de pacientes, a estratégia de monoterapia ainda pode ser indicada. Pacientes portadores de hipertensão portal, HIV, cardiopatia congênita complexa, doença veno-oclusiva ou aqueles com HAPI e alta probabilidade de insuficiência cardíaca esquerda, com fração de ejeção preservada, podem iniciar tratamento utilizando apenas uma classe de fármaco. Além disso, pacientes que são já usuários de monoterapia e estejam em estabilidade clínica por longos períodos não necessitam obrigatoriamente de combinação farmacológica. ${ }^{23}$

O potencial da combinação de três fármacos desde o diagnóstico também já foi avaliado. Um estudo retrospectivo francês avaliou a eficácia da combinação tripla (epoprostenol, bosentana e sildenafil) no tratamento inicial de pacientes recém-diagnosticados com formas graves de HAP, o que demonstrou melhora na classe funcional, na capacidade de exercício e nos parâmetros hemodinâmicos. O estudo mostrou ainda que a sobrevida dos pacientes com terapia tripla foi melhor do que a prevista pelo registro histórico francês, sugerindo que exista benefício a longo prazo com o uso desta abordagem. ${ }^{49}$

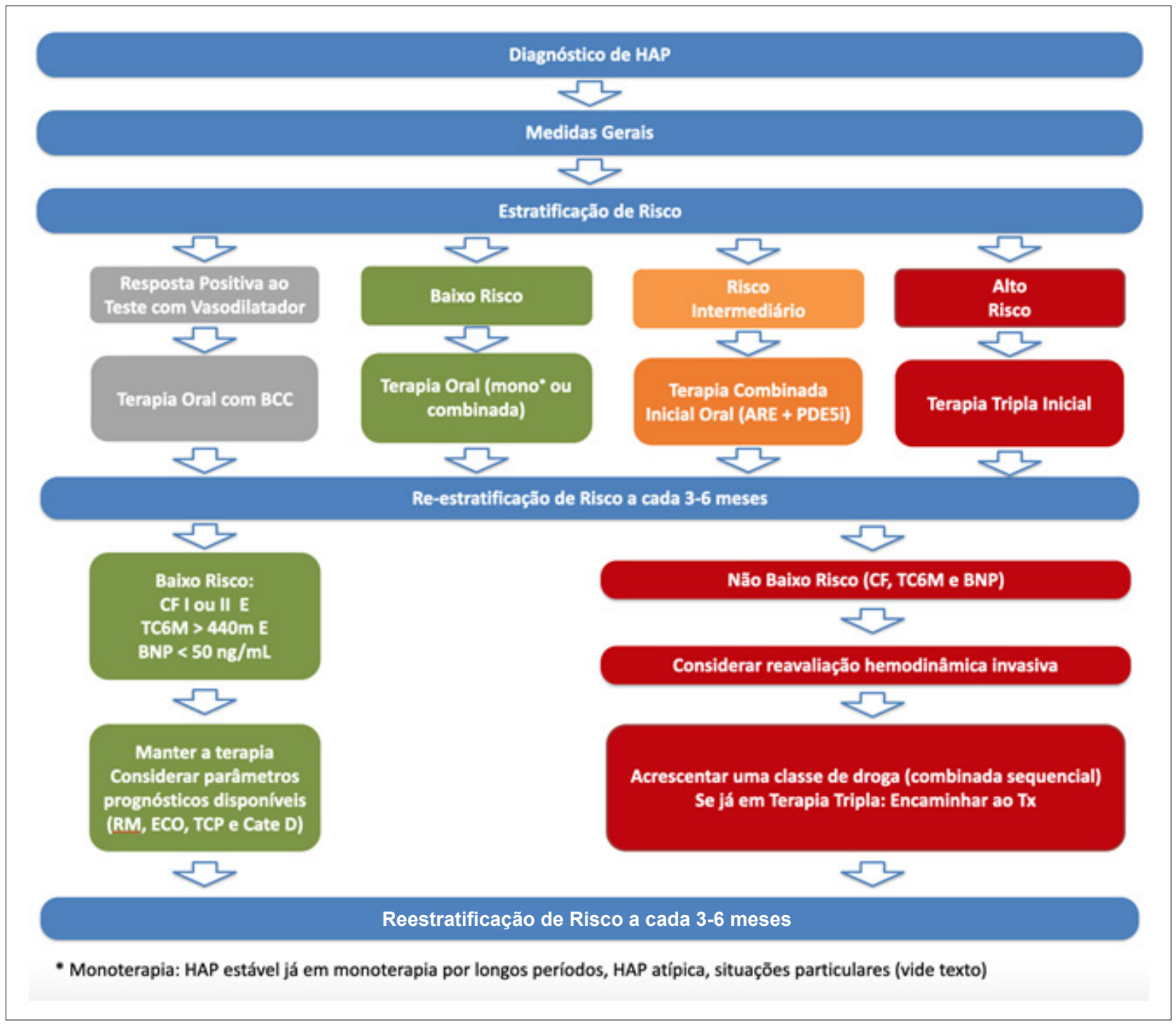

Figura 2 - Algoritmo de tratamento e estratificação de risco no seguimento da HAP realizado pelo grupo de circulação pulmonar do Incor (USP). 
No entanto, para pacientes menos graves, não há evidência que comprove a eficácia da terapia tripla inicial. Um estudo recente avaliou o uso de selexipague, macitentana e tadalafil versus terapia dupla inicial com macitentana e tadalafil em 247 pacientes recém-diagnosticados com HAP. ${ }^{50}$ Não foi detectada diferença na melhora da RVP, na distância percorrida no teste de caminhada de 6 minutos (TC6M) e nos níveis de NTproBNP. Além disso, efeitos adversos foram mais presentes nos pacientes em uso de terapia tripla.

Por outro lado, a combinação sequencial de três fármacos para o tratamento de HAP parece ser eficiente. A adição do selexipague ao tratamento já estabelecido de ARE e PDE5i foi associada à diminuição do número de hospitalizações e eventos de progressão de doença. ${ }^{51}$ $\mathrm{O}$ benefício identificado foi mais pronunciado em pacientes em classe funcional 2 .

Outra potencial estratégia seria a substituição de medicações dentro de uma mesma via, caso o paciente não apresente resposta clínica satisfatória. Apesar de promissora, ela ainda carece de comprovação científica robusta e não deve ser realizada de forma rotineira. ${ }^{52}$

\section{Estratificação de risco e seguimento clínico}

Avaliar o risco de progressão da HAP é fundamental para orientar seu tratamento. Estratégias de estratificação de risco de morte, avaliando a combinação de múltiplos marcadores (Tabela 3) tanto ao diagnóstico quanto ao longo do tratamento, mostraram-se eficientes para predizer o curso clínico da doença. ${ }^{53-55}$
Existem várias formas de avaliar o risco de progressão da HAP. Uma delas é através da atribuição de 1 a 3 pontos para cada variável, de acordo com a faixa de risco em que ela se encontra (baixo, intermediário ou alto, respectivamente). Para obtenção do risco global, divide-se a soma de todos os pontos pelo número de variáveis utilizadas. O número inteiro mais próximo determina o risco em baixo (1), intermediário (2) ou alto (3). ${ }^{55}$ Outra abordagem, mais simples, procura apenas identificar os pacientes que possam ser classificados como baixo risco, através da combinação dos seguintes parâmetros combinados: níveis de BNP inferiores a $50 \mathrm{pg} / \mathrm{mL}$, TC6M superior a $440 \mathrm{~m}$ e CF menor ou igual a 2.53 Existe ainda uma terceira abordagem, derivada da calculadora de risco oriunda do registro americano REVEAL (Registry to Evaluate Early and Long-Term PAH Disease Management), baseada em até 12 variáveis e que foi recentemente atualizada. ${ }^{56}$

Embora seja claro que a avaliação mais abrangente possa ter superioridade em relação à avaliação simplificada, a disponibilidade dos diferentes exames necessários limita sua utilização. Dessa forma, a estratificação de risco aqui sugerida baseia-se no que deve ser considerado o mínimo necessário para o adequado manejo clínico dos pacientes com HAP, através da combinação entre CF, TC6M e BNP, para a identificação de baixo risco ou não baixo risco (Figura 2). Conforme disponibilidade, exames de ressonância magnética, ecocardiograma e teste cardiopulmonar de esforço devem ser considerados, haja vista seu potencial papel adjuvante. ${ }^{23,57}$ O objetivo do tratamento da HAP é fazer com que o paciente permaneça ou atinja baixo risco de progressão de doença. Para isso, as diferentes estratégias já discutidas são utilizadas de acordo com a estratificação de risco encontrada.

Tabela 3 - Fatores prognósticos na hipertensão arterial pulmonar (adaptada da referência 8)

\begin{tabular}{|c|c|c|c|}
\hline \multirow{2}{*}{ Determinantes do prognóstico } & \multicolumn{3}{|c|}{ Mortalidade estimada em 1 ano } \\
\hline & Baixo risco: $<5 \%$ & Risco intermediário: 5-10\% & Alto risco: $>10 \%$ \\
\hline $\begin{array}{l}\text { Sinais clínicos de insuficiência } \\
\text { ventricular direita }\end{array}$ & Ausente & Ausente & Presente \\
\hline Progressão dos sintomas & Não & Lenta & Rápida \\
\hline Síncope & Não & Ocasionalmente ${ }^{*}$ & Repetidamente ${ }^{* *}$ \\
\hline CF OMS & I, II & III & IV \\
\hline DC6M & $>440 \mathrm{~m}$ & $165-440 \mathrm{~m}$ & $<165 \mathrm{~m}$ \\
\hline Teste de exercício cardiopulmonar & $\begin{array}{c}\text { Pico VO }>15 \mathrm{~mL} / \mathrm{min} / \mathrm{kg} \\
(>65 \% \text { pred.) } \\
\text { Alça VE } / \mathrm{NCO}_{2}<36\end{array}$ & $\begin{array}{c}\text { Pico VO } 11-15 \mathrm{~mL} / \mathrm{min} / \mathrm{kg} \\
(35-65 \% \text { pred.) } \\
\text { Alça VE } / \mathrm{NCO}_{2} 36-44,9\end{array}$ & $\begin{array}{c}\text { Pico } \mathrm{VO}_{2}<11 \mathrm{~mL} / \mathrm{min} / \mathrm{kg} \\
(<35 \% \text { pred.) } \\
\mathrm{VE} \mathrm{NCO}_{2} \geq 45\end{array}$ \\
\hline $\begin{array}{l}\text { Níveis plasmáticos } \\
\text { de NT-proBNP }\end{array}$ & $\begin{array}{c}\text { BNP }<50 \mathrm{ng} / \mathrm{L} \\
\text { NT-proBNP}<300 \mathrm{ng} / \mathrm{mL}\end{array}$ & $\begin{array}{l}\text { BNP } 50-300 \text { ng/L } \\
\text { NT-proBNP } 300-1.400 \text { ng/L }\end{array}$ & $\begin{array}{c}\text { BNP > } 300 \mathrm{ng} / \mathrm{L} \\
\text { NT-proBNP }>1.400 \mathrm{ng} / \mathrm{L}\end{array}$ \\
\hline $\begin{array}{l}\text { Exames de imagem (ECO, ressonância } \\
\text { magnética do tórax) }\end{array}$ & $\begin{array}{l}\text { Área do } A D<18 \mathrm{~cm}^{2} \\
\text { Ausência de derrame pericárdico }\end{array}$ & $\begin{array}{c}\text { Área do AD } 18-26 \mathrm{~cm}^{2} \\
\text { Ausência ou mínimo derrame } \\
\text { pericárdico }\end{array}$ & $\begin{array}{l}\text { Área do } A D>26 \mathrm{~cm}^{2} \\
\text { Derrame pericárdico presente }\end{array}$ \\
\hline Parâmetros hemodinâmicos & $\begin{array}{c}\text { Pressão do } \mathrm{AD}<8 \mathrm{mmHg} \\
\mathrm{IC} \geq 2,5 \mathrm{~L} / \mathrm{min} / \mathrm{m}^{2} \\
\mathrm{SvO}_{2}>65 \%\end{array}$ & $\begin{array}{c}\text { Pressão do AD } 8-14 \mathrm{mmHg} \\
\text { IC } 2,0-2,4 \mathrm{~L} / \mathrm{min} / \mathrm{m}^{2} \\
\mathrm{SvO}_{2} 60-65 \%\end{array}$ & $\begin{array}{c}\text { Pressão do } \mathrm{AD}>14 \mathrm{mmHg} \\
\mathrm{IC}<2,0 \mathrm{~L} / \mathrm{min} / \mathrm{m}^{2} \\
\mathrm{SvO}_{2}<60 \% \\
\end{array}$ \\
\hline
\end{tabular}

* Síncope ocasional durante exercício brusco ou intenso, ou síncope ortostática ocasional em paciente previamente estável. ** Episódios repetidos de síncope, mesmo em atividade física leve ou regular.

$V_{2}$ pico: consumo de oxigênio no pico do esforço; VE: ventilação; $V_{C O}$; volume expirado de $\mathrm{CO}_{2}$; $B N P / N T$-próBNP: peptídeo natriurético tipo B/fragmento $\mathrm{N}$-terminal do peptídeo natriurético tipo $\mathrm{B} ; \mathrm{AD}$ : átrio direito; IC: índice cardíaco; $\mathrm{SvO}_{2}$ : saturação venosa central de oxigênio. 


\section{Particularidades das diversas formas de HAP}

\section{HAP associada às doenças do tecido conjuntivo}

A HAP é uma complicação conhecida em pacientes com doença do tecido conjuntivo (DTC). As principais DTCs associadas à HAP são esclerose sistêmica (ES), lúpus eritematoso sistêmico (LES) e doença mista do tecido conjuntivo (DMTC); em menor grau, dermatomiosite e síndrome de Sjögren. ${ }^{58}$ No Brasil, a HAP associada à DTC representa cerca de $25 \%{ }^{59}$ dos casos de HAP. Nos países ocidentais, a ES é a principal doença associada à ocorrência de HAP, enquanto em países asiáticos é o LES. ${ }^{60-62}$

A prevalência de HAP em pacientes com ES é em torno de $10 \%$, ${ }^{63}$ e no LES é de $4 \%{ }^{64}$ A presença de anticorpos antifosfolípideos, anti-RNP e anti-Ro são preditivos de HAP em pacientes com LES. ${ }^{65}$ Em contrapartida, em pacientes com ES, doença de longa evolução, presença de telangiectasias, positividade do anticentrômero e redução de DLCO são os principais fatores relacionados à HAP. ${ }^{66}$

A ES é uma doença representada pela associação de vasculopatia e fibrose tecidual. ${ }^{67}$ Clinicamente, apresenta uma alta frequência de doença pulmonar intersticial (até $50 \%$ dos pacientes) ${ }^{68}$ e acometimento cardíaco (50 a 80\% dos pacientes), ocasionando, frequentemente, disfunção diastólica. ${ }^{69}$ Portanto, nestes pacientes, a HP pode ser resultado de uma doença vascular pulmonar isolada ou associada aos diferentes mecanismos fisiopatológicos relacionados à HP dos grupos 2 e $3 .{ }^{68}$ Além disso, esses pacientes possuem risco aumentado de trombembolismo pulmonar e também podem apresentar doença veno-oclusiva associada, impactando ainda mais o seu prognóstico. ${ }^{68}$
É imperativo tentar determinar qual é o mecanismo preponderante, pois é o fator que ditará o tratamento.

Em pacientes com ES, é recomendada triagem anual de $\mathrm{HP}$, mesmo nos assintomáticos. ${ }^{70}$ Os modelos de triagem compostos com fatores de risco, DLCO e BNP parecem ser mais sensíveis do que o uso isolado do ecocardiograma. ${ }^{71} \mathrm{No}$ LES, diante da baixa prevalência de HAP, o rastreamento não é recomendado como rotina, devendo o ecocardiograma ser realizado apenas na presença de sintomas (Tabela 4).

Diante da suspeita clínica, o fluxograma diagnóstico é o mesmo, objetivando confirmar HAP (grupo 1) e descartar outras formas de HP. O teste de vasorreatividade não é aplicado aos pacientes com DTC, uma vez que menos de $1 \%$ apresentam resposta sustentada.

Nos pacientes com formas mais inflamatórias como LES ou DMTC-HAP, pode optar-se pela imunossupressão antes do início de terapia específica. Em pacientes em classes funcionais I e II, recomenda-se o início de tratamento apenas com ciclofosfamida (CFF) e glicocorticoide (GCT). Nos casos em classes funcionais III e IV, a chance de resposta apenas com imunossupressores é menor, e recomenda-se uso de CFF e GCT associados à terapia específica, com reavaliação após 6 meses de CFF (Tabela 5). ${ }^{58,72}$ Nos pacientes com ES, não há evidência de melhora com uso de imunossupressor. ${ }^{73}$

\section{HAP associada ao HIV}

A associação entre HIV e HAP foi descrita pela primeira vez em 1987. ${ }^{74}$ Atualmente, estima-se uma prevalência de $0,5 \%$ de HAP nos pacientes portadores de $\mathrm{HIV}^{75}$ sendo uma etiologia bastante relevante nos centros de referência. Em um registro brasileiro, 4,5\% dos pacientes com HAP eram portadores de HIV. ${ }^{59}$

Tabela 4 - Fatores de risco e rastreamento de HAP em pacientes com doença do tecido conjuntivo

\begin{tabular}{|c|c|c|}
\hline & Fator de risco & Rastreamento \\
\hline ES & $\begin{array}{c}\text { Doença de longa evolução } \\
\text { Telangiectasias } \\
\text { Anticentrômero } \\
\text { Redução de DLCO ou relação CVF/DLCO>1.6 }\end{array}$ & $\begin{array}{c}\text { Anual em assintomáticos } \\
\text { ECO } \pm \text { biomarcadores/ PFP ou algoritmo DETECT } \\
\text { BNP/NT-próBNP e PFP } \pm \text { ECO }\end{array}$ \\
\hline LES & $\begin{array}{c}\text { Serosite } \\
\text { Anticorpos: antifosfolípideos, RNP e Ro }\end{array}$ & Apenas em sintomáticos \\
\hline
\end{tabular}

ES: esclerose sistêmica; LES: lúpus eritematoso sistêmico; PFP: Prova de função pulmonar; DLCO: capacidade de difusão pulmonar para o monóxido de carbono; capacidade vital forçada (CVF); BNP/NT-próBNP: peptídeo natriurético tipo B/fragmento N-terminal do peptídeo natriurético tipo $B$.

Tabela 5 - Tratamento da HAP associada às doenças do tecido conjuntivo

\begin{tabular}{lcc}
\hline & Imunossupressão (IS) & Terapia específica \\
\hline ES & Não recomendada & Conforme estratificação de risco \\
\hline LES classe funcional III/IV & CFF e GCT por 6 meses & Quando mantido HP após IS \\
\hline DMTC classe funcional I/II & $\begin{array}{c}\text { CFF e GCT associada } \\
\text { à terapia específica }\end{array}$ & Associada a IS, conforme estratificação de risco \\
\hline DMTC classe funcional III/IV & CFF e GCT por 6 meses & Quando mantido HP após IS \\
\hline
\end{tabular}

ES: esclerose sistêmica; LES: lúpus eritematoso sistêmico; DMTC: doença mista do tecido conjuntivo CFF: ciclofosfamida; GCT: glicocorticoide ; HP: hipertensão pulmonar. 
Os mecanismos fisiopatológicos envolvidos no quadro de HIV e HAP ainda não estão totalmente esclarecidos. Do ponto de vista histológico, em até $78 \%$ dos pacientes foi observado fibrose concêntrica da íntima, hipertrofia da média e lesões plexiformes, algo muito semelhante as causas não infeciosas de $\mathrm{HAP}^{76}$

A presença de HAP confere pior prognóstico ao paciente com HIV, tendo a contagem de CD4 ${ }^{+}$menor que 200 células/ $\mu \mathrm{L}$ e débito cardíaco menor que $2,8 \mathrm{~L} / \mathrm{min}$ por $\mathrm{m}^{2}$ como preditores independentes de sobrevida. ${ }^{77}$ Recomenda-se, portanto, o tratamento com a terapia antirretroviral (TARV) para todos os pacientes com HIV, independente da contagem de $\mathrm{CD}^{+}{ }^{+}$e carga viral, ${ }^{78}$ e da HAP concomitante.

Considerando a via do óxido nítrico, não existem estudos controlados com sildenafil que avaliem a resposta ao tratamento nos pacientes com HIV-HAP, e os resultados positivos encontrados com capacidade de exercício, CF e hemodinâmica derivam de estudos de casos. ${ }^{79}$ Sugere-se cautela, devido à interação com a TARV, especialmente com os inibidores de protease (IPr). ${ }^{80}$ Também não há estudos específicos com o riociguate, apesar de publicação recente que demonstrou segurança e tolerabilidade da associação entre este fármaco e a TARV. ${ }^{81}$

Entre os AREs, a bosentana foi avaliada para o tratamento de portadores de HVI-HAP em um estudo prospectivo com 59 pacientes. ${ }^{82}$ Foi demonstrado melhora no TC6M, nos sintomas e em dados hemodinâmicos. Nos estudos pivotais que avaliaram o uso de ambrisentana ${ }^{40}$ e macitentana, ${ }^{39}$ foram incluídos 17 e 10 pacientes com HIV-HAP, respectivamente, e não se encontraram interações com a TARV. ${ }^{78}$

Pequenas séries de casos avaliaram alguns prostanoides para o tratamento de HIV-HAP. O epoprostenol melhorou os dados hemodinâmicos em 6 pacientes. ${ }^{83} \mathrm{O}$ iloprost mostrou efeito na melhora da CF e do TC6M de 4 pacientes. ${ }^{84} \mathrm{O}$ selexipague foi administrado em 10 pacientes portadores de HIV no seu estudo pivotal. ${ }^{35}$ Não houve interação de selexipague com a TARV, nem necessidade de mudança de dose em um estudo de pacientes saudáveis em uso de selexipague 400 mcg uma vez ao dia e de dois antirretrovirais (lopinavir/ritonavir). ${ }^{85}$

\section{Hipertensão portopulmonar}

A hipertensão portopulmonar (HPOP) é uma forma de HAP que surge na vigência de hipertensão portal que gera a abertura de shunts portossistêmicos e um aumento do fluxo sanguíneo na circulação pulmonar. Esse hiperfluxo acarreta disfunção e remodelamento endotelial, com aumento da produção de ET1 e elevação da pressão no território vascular pulmonar. ${ }^{86}$

Estima-se que aproximadamente $10,6 \%$ dos pacientes com HAP possuem HPOP ${ }^{87}$ Por outro lado, HPOP está presente em $8,5 \%$ dos pacientes avaliados para transplante hepático. ${ }^{88}$ A HPOP não possui relação com a etiologia de hipertensão portal ou com a gravidade da doença hepática apresentada. ${ }^{89}$

A apresentação clínica dos pacientes é semelhante a outras formas de HAP, e o sintoma mais frequente é a dispneia. A ascite em paciente com HAP pode ser indicativa da presença de HPOP. ${ }^{90} \mathrm{O}$ diagnóstico dessa condição envolve a realização de cateterismo direito para a confirmação de HAP, conforme previamente descrito, e a confirmação de hipertensão portal, via medida do gradiente de pressão trans-hepática superior a $4 \mathrm{mmHg} .{ }^{91}$

O tratamento de HPOP foi avaliado através de pequenos estudos, que demonstraram benefício das três classes de fármacos alvo para HAP. A hepatotoxicidade dos AREs não parece ser um problema maior para essa população, desde que monitorizados adequadamente. ${ }^{92} \mathrm{O}$ maior estudo realizado em pacientes com HPOP demonstrou melhora hemodinâmica após 12 semanas de uso de macitentana, sem efeitos colaterais hepáticos. ${ }^{93}$ Alguns fármacos bem estabelecidos para o tratamento de hipertensão portal, como os betabloqueadores, não devem ser administradas em HPOP, por gerarem piora hemodinâmica e na capacidade de exercício. ${ }^{94}$

Vale a pena ressaltar o papel da HPOP nos pacientes em avaliação para transplante hepático. A HPOP é um marcador de pior prognóstico perioperatório neste procedimento. ${ }^{91}$ Valores de PMAP maiores do que $50 \mathrm{mmHg}$ contraindicam o transplante, e menores que $35 \mathrm{mmHg}$ indicam baixo risco de morte na realização do procedimento. ${ }^{91}$ Dessa forma, é fundamental a realização de um ecocardiograma para rastreamento de HAP em pacientes na fila para transplante hepático, ou em pacientes com hipertensão portal e dispneia, mesmo que de pequena intensidade. ${ }^{95}$

\section{Esquistossomose}

A esquistossomose é uma doença parasitária endêmica no Brasil, relacionada a pobreza e más condições sanitárias, sendo causada por um verme trematódeo do gênero Schistosoma, cuja transmissão já foi relatada em mais de 70 países. A manifestação mais comum da esquistossomose crônica é a sua forma hepatoesplênica. ${ }^{96}$ No entanto, uma das suas manifestações mais graves e limitantes advém do acometimento da circulação pulmonar, com a presença de HAP.

Cerca de $5 \%$ dos pacientes com a forma hepatoesplênica da esquistossomose apresentam HAP. ${ }^{96,97}$ Assim, dada a alta prevalência mundial da esquistossomose, a HAP-Sch é potencialmente uma das formas mais prevalentes de HAP no mundo, particularmente em países emergentes. ${ }^{97} \mathrm{Um}$ registro recente demonstrou que a HAP-Sch pode representar cerca de $20 \%$ dos casos incidentes de HAP. ${ }^{59}$

O diagnóstico de HAP-Sch requer a confirmação invasiva de HAP (vide a sessão definições) e de esquistossomose hepatoesplênica, mediante a presença de alterações ultrassonográficas compatíveis com a doença (presença de fibrose periportal), mais um dos três fatores epidemiológicos que associem a esquistossomose à condição ultrassonográfica encontrada: 1) paciente proveniente de área endêmica para a doença; 2) tratamento prévio para esquistossomose; e 3) presença de ovos do parasita no exame de fezes ou na biópsia retal..$^{98}$

A HAP-Sch possui melhor curso clínico que a HAPI, mesmo na ausência de terapia específica; entretanto, isso não faz com que a doença seja isenta de risco. A taxa de mortalidade associada a HAP-Sch pode chegar até 15\% em 3 anos. ${ }^{99}$ Casos de HAP com pronunciada dilatação das artérias pulmonares 
devem suscitar a hipótese de HAP-Sch, principalmente em regiões altamente prevalentes para essa condição. ${ }^{100}$

O tratamento de HAP-Sch é bastante semelhante ao de outras formas de HAP. Dados coletados em séries de casos demonstraram benefícios clínicos, hemodinâmicos ${ }^{101}$ e de sobrevida com o tratamento específico; ${ }^{97}$ assim, recomenda-se também seguir o algoritmo de tratamento proposto na Figura 2 para esta forma de HAP. A anticoagulação deve ser evitada, pelo risco de hemorragia digestiva. Todos os pacientes devem receber pelo menos um ciclo do tratamento parasitário, já que não se sabe se a persistência da infecção pode contribuir com a progressão do quadro vascular pulmonar. ${ }^{97}$

\section{Cardiopatia congênita}

A HAP é uma condição relativamente frequente nos pacientes portadores de cardiopatias congênitas (CG), acometendo de 5-10\% desta população. ${ }^{102}$ Tais cardiopatias podem gerar hiperfluxo na circulação pulmonar, induzindo a remodelamento endotelial e consequente da RVP, histologicamente indistinguível de outras formas de HAP. Dessa forma, a maioria das situações de HAP-CG são classificadas como grupo 1. Nesse grupo, quando a RVP supera a RVS e há a inversão do fluxo sanguíneo pelo shunt, caracteriza-se a síndrome de Eisenmenger. ${ }^{103}$ Esses pacientes possuem hipoxemia grave e, consequentemente, alterações hematológicas (eritrocitose e plaquetopenia). Pacientes com Eisenmenger também podem apresentar hemoptise, acidente vascular cerebral (AVC), abscessos cerebrais e maior incidência de morte súbita. ${ }^{9}$

O tratamento de HAP-CG segue as orientações previamente descritas para outras formas do grupo 1. Quando os sintomas de hiperviscosidade estiverem presentes na síndrome de Eisenmenger (geralmente com hematócrito $>65 \%$ ), a flebotomia terapêutica pode ser realizada. ${ }^{9}$ Assim como em outras formas de HAP, a anticoagulação na síndrome de Eisenmenger é controversa, ${ }^{104}$ devendo ser ponderada caso a caso. O benefício do uso de oxigênio suplementar em pacientes com síndrome de Eisenmenger e hipoxemia é questionável, ${ }^{105}$ e sua indicação deve ser feita de forma individualizada.

Do ponto de vista de tratamento específico, o único fármaco validado de forma prospectiva na HAP-CG foi a bosentana, que melhorou o TC6M e diminui a RVP após 16 semanas de tratamento em pacientes com CF III. ${ }^{106}$ Séries menores demonstraram benefício com PDE5i e prostanoides. ${ }^{9}$

Algumas CGs como patência do ducto arterioso, defeito do septo interatrial do tipo seio venoso ou anormalidades de drenagem das veias pulmonares podem passar despercebidas em pacientes com HAP e serem erroneamente classificados como HAPI. No entanto, a identificação das CGs no contexto de HAP pode indicar a necessidade de correção cirúrgica do defeito, a depender da RVP ao diagnóstico ${ }^{107}$ e do comportamento clínico. Esse tipo de avaliação deve ser feito em centros com experiência nessa condição, a fim de indicar adequadamente a correção ou não. Apenas a possibilidade de correção já ressalta o quão fundamental é investigar ativamente a presença de CG. Uma estratégia de investigação das CGs em pacientes sob avaliação diagnóstica de HAP é a realização de ecocardiograma com microbolhas, buscando shunts intra e extracardíacos.

Algumas cardiopatias congênitas complexas, mais raras, são classificadas como grupo 5, de mecanismos incertos ou multifatorial. ${ }^{8}$ Nesses casos, dada a ausência e estudos prospectivos a abordagem farmacológica ou cirúrgica (incluindo transplantes de coração-pulmão) deve ser realizada de forma individualizada.

\section{Situações especiais}

\section{Hipertensão pulmonar e gestação}

As mudanças fisiológicas ao longo da gestação, como o aumento do volume sanguíneo e do DC, ${ }^{108,109}$ geralmente são mal toleradas pelas pacientes com HAP. Dessa maneira, a gravidez nessa condição é associada a elevadas taxas de morbimortalidade, oscilando entre 30 a $56 \%{ }^{18}$

Dada a alta letalidade da HAP em gestantes, recomenda-se a utilização de dois métodos contraceptivos. Não há consenso sobre o melhor método: os de barreira são seguros, porém a eficácia é diretamente relacionada ao uso adequado. ${ }^{108}$ Anticoncepcionais hormonais com progesterona isolada são efetivos, evitando a associação aos estrógenos, que podem aumentar os riscos de eventos trombembólicos. ${ }^{109}$ Dispositivos intrauterinos são uma opção, mas podem levar a reações vasovagais no momento da inserção. ${ }^{110}$

Caso a gestação ocorra, a mulher deve ser informada sobre os riscos potenciais. Em casos mais extremos, podese, inclusive, considerar a possibilidade de interrupção terapêutica da gestação. ${ }^{109}$ Caso esta seja a decisão, o aborto deve ser realizado preferencialmente até a $22^{\underline{a}}$ semana. ${ }^{111} \mathrm{Em}$ um registro europeu recente de HP e gestação, ${ }^{112}$ o aborto terapêutico foi realizado em $4 \%$ dos casos; em contrapartida, a mortalidade perinatal nas pacientes com HAPI foi de $43 \%$. Não há consenso sobre a melhor via de parto, mas há a tendência em realizar a cesariana entre 32 a 36 semanas, ${ }^{111}$ evitando-se a anestesia geral. ${ }^{113}$ Pré-eclampsia ou eclampsia, parto prematuro, morte fetal e sangramento pós-parto foram as complicações mais frequentes nas gestantes com HP. ${ }^{114}$

O tratamento específico de HAP deve ser adaptado caso a paciente engravide e opte pela manutenção da gestação até o termo. Algumas séries de casos demonstraram eficácia na manutenção do uso de sildenafil e análogos da PGI2 na gestação. ${ }^{18,113,115}$ No entanto, os AREs devem ser evitados por conta de seu potencial efeito teratogênico. ${ }^{116}$

\section{Angina e HAP}

A angina é referida por $15,8 \%{ }^{117}$ a $29 \%{ }^{9}$ dos pacientes com HAP, e pode decorrer do desequilíbrio entre a oferta e o consumo miocárdico de oxigênio no ventrículo direito, sem alteração de fluxo em coronárias epicárdicas. O aumento da tensão da parede ventricular acarreta redução da reserva de fluxo coronária, o que associado ao maior consumo de oxigênio pelo ventrículo, com aumento do seu trabalho e hipertrofia miocárdica, já justificaria a isquemia. Entretanto, outra consequência da HAP é o remodelamento vascular, com aumento do diâmetro de artérias pulmonares. 
Por sintopia, a artéria pulmonar dilatada e com regime hipertensivo pode determinar deslocamento inferior do tronco da artéria coronária esquerda (TCE) que se aproxima do seio coronário esquerdo (Figura 3). Essa compressão pode acarretar importante diminuição de calibre do óstio do TCE. ${ }^{118}$ Em pacientes com angina e HAP, é descrita prevalência de até $40 \%$ de obstrução significativa em TCE por compressão extrínseca. ${ }^{117} \mathrm{O}$ maior preditor de compressão de TCE nesses pacientes foi o diâmetro de artéria pulmonar $>40 \mathrm{~mm}$, seguido da relação artéria pulmonar/aorta $\geq 1,5 .{ }^{117}$

O impacto prognóstico da compressão do TCE na HAP ainda é incerto. O manejo terapêutico dessa compressão é feito analogamente ao que se faz em lesão de TCE por aterosclerose: a revascularização miocárdica deve ser considerada, sendo a abordagem percutânea a modalidade mais atrativa e segura para esses pacientes. ${ }^{119}$ Dados italianos demonstraram um bom resultado com essa abordagem. Entre 53 pacientes submetidos a angioplastia e com tempo médio de seguimento de 4,5 anos, 19 faleceram $(37,3 \%)$ sem nenhum caso de infarto ou trombose de stent, sendo que 5 pacientes necessitaram nova angioplastia. ${ }^{120}$

A compressão de TCE deve ser considerada em todos os pacientes com hipertensão pulmonar e angina, com investigação inicial pela angiotomografia coronária. Pacientes em programação de realizar cateterismo direito e com alta probabilidade clínica de obstrução extrínseca de TCE (por exemplo, angina em pacientes com grandes aneurismas de artéria pulmonar) podem fazer a cineangiocoronariografia diretamente, mesmo sem a angiotomografia de coronárias. Ressalta-se que, diante do aumento da sobrevida em pacientes com HAP, a comorbidade aterosclerótica também deve ser considerada, sendo importante o controle dos fatores tradicionais de risco cardiovascular.

\section{Arritmias supraventriculares e HAP}

As arritmias cardíacas são frequentes em pacientes com HAP; notadamente as taquicardias supraventriculares, quer por exacerbação de automatismo, quer por mecanismos de reentrada. Estudos retrospectivos reportam incidência anual de taquicardia supraventricular sustentada entre $2,8 \%{ }^{121} \mathrm{e}$ $3,5 \%{ }^{122}$ entretanto, tais taxas nitidamente subestimam a real incidência, dada a falta de estudos prospectivos com busca sistemática, e estratégia de monitorização contínua ou de demanda acessível.

A sobrecarga pressórica em câmaras direitas, o remodelamento cardíaco e o elevado tônus adrenérgico associados a hipertensão pulmonar propiciam essas arritmias. Hipocalemia e hipomagnesemia, comumente desencadeadas pelo uso crônico de diuréticos, também podem ser gatilhos.

As arritmias sustentadas mais comuns são a fibrilação atrial e o flutter atrial; porém, taquicardia por reentrada nodal e taquicardia atrial sustentada também são observadas. A maioria dos pacientes apresenta piora de classe funcional com os episódios de arritmia, e a persistência da arritmia confere maior mortalidade. ${ }^{121} \mathrm{O}$ tratamento tem como alvo preferencial a restauração do ritmo cardíaco por meio do uso de fármacos de pouca influência inotrópica (como a amiodarona), cardioversão elétrica programada e, em casos selecionados, estudo eletrofisiológico e ablação. Esta última técnica deve ser precocemente considerada para os pacientes com flutter atrial. No preparo para cardioversão, ou em casos de recorrência e falha da mesma, o controle da resposta ventricular é fundamental para assegurar o débito cardíaco, devendo-se individualizar a terapia de acordo com a reserva funcional do ventrículo direito. Pacientes com disfunção importante de ventrículo direito por hipertensão pulmonar toleram mal betabloqueadores, e mesmo o uso de bloqueadores de canal de cálcio podem causar descompensação clínica. Nessas situações limítrofes, a utilização de digoxina e da própria amiodarona se torna uma opção. Cabe ainda ressaltar que todo paciente com hipertensão pulmonar e fibrilação atrial, ou flutter, precisa receber terapia anticoagulante, pois o risco de trombembolismo sistêmico e cerebral é alto.

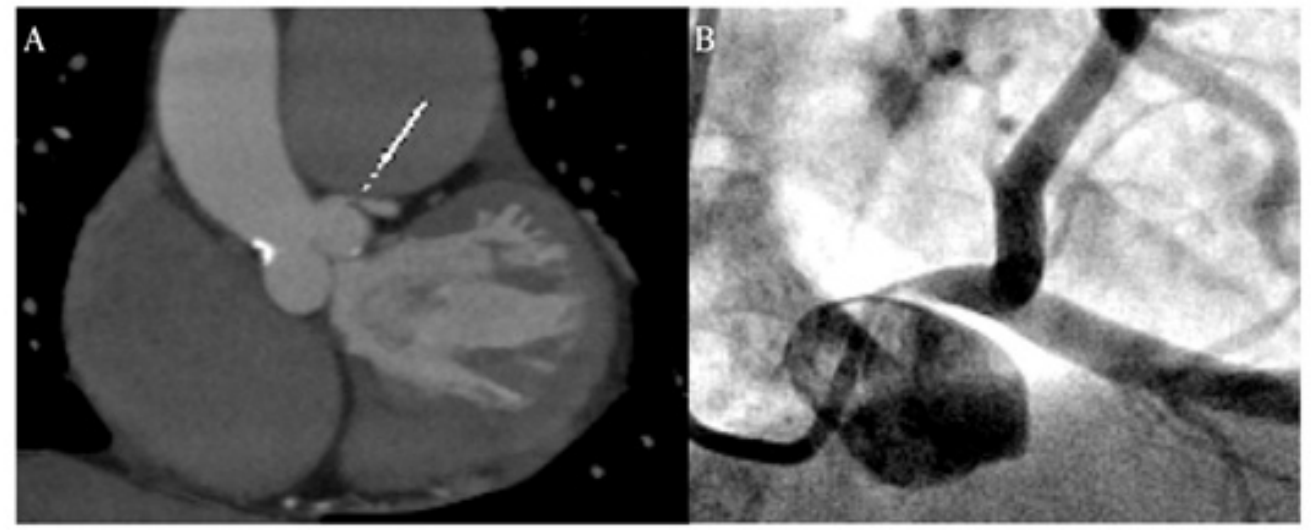

Figura 3 - A) Angiotomografia coronária evidenciando deslocamento inferior do tronco da artéria coronária esquerda (TCE) por artéria pulmonar aneurismática e redução do calibre do óstio do TCE. B) Cineangiocoronariografia do mesmo paciente, evidenciando a suboculsão do óstio do TCE. 


\section{Conclusão}

Muitos avanços ocorreram nas últimas décadas na terapêutica da HAP, uma doença grave, progressiva, incurável e potencialmente fatal. Para seu tratamento adequado, é fundamental o diagnóstico hemodinâmico e a classificação da sua etiologia. Várias etiologias (colagenoses, hipertensão portal, cardiopatia congênitas, esquistossomose) requerem medidas específicas, além do tratamento farmacológico específico para HAP.

O tratamento específico para HAP baseia-se em fármacos que interferem em três vias fisiopatológicas: da prostaciclina, a da endotelina e a do óxido nítrico. Atualmente, recomendase que o tratamento inicial inclua uma combinação de duas terapias orais e que seja aumentado caso o paciente não alcance os alvos terapêuticos desejados, estes determinados através da estratificação de risco de morte cardiovascular. Complicações cardiovasculares de HAP (compressão de tronco de coronária esquerda, arritmias supraventriculares) são frequentes e devem ser prontamente identificadas e tratadas, já que impactam a qualidade de vida e, potencialmente, o prognóstico de pacientes com HAP.

\section{Contribuição dos autores}

Concepção e desenho da pesquisa: Fernandes CJ, Calderaro D; Obtenção de dados e Redação do manuscrito:

\section{Referências}

1. Humbert M. Pulmonary Arterial Hypertension and Chronic Thromboembolic Pulmonary Hypertension: Pathophysiology. Eur Respir Rev. 2010;19(115):59-63. doi: 10.1183/09059180.00007309.

2. Alves JL Jr, Oleas FG, Souza R. Pulmonary Hypertension: Definition, Classification, and Diagnosis. Semin Respir Crit Care Med. 2017;38(5):561570. doi: 10.1055/s-0037-1606577.

3. Hoeper MM, Humbert M, Souza R, Idrees M, Kawut SM, Sliwa-Hahnle K, et al. A global View of Pulmonary Hypertension. Lancet Respir Med. 2016;4(4):306-22. doi: 10.1016/S2213-2600(15)00543-3.

4. D'Alonzo GE, Barst RJ, Ayres SM, Bergofsky EH, Brundage BH, Detre KM, et al. Survival in Patients with Primary Pulmonary Hypertension. Results from a National Prospective Registry. Ann Intern Med. 1991;115(5):343-9. doi: 10.7326/0003-4819-115-5-343.

5. Jardim C, Fernandes CJ, Souza R. Goal-Oriented Treatment of Pulmonary Arterial Hypertension. Curr Opin Pulm Med. 2014;20(5):409-13. doi: 10.1097/MCP.0000000000000086.

6. Fernandes CJ, Martins BC, Jardim CV, Ciconelli RM, Morinaga LK, Breda AP, et al. Quality of Life as a Prognostic Marker in Pulmonary Arterial Hypertension. Health Qual Life Outcomes. 2014;12(1):130. doi: 10.1186/ s12955-014-0130-3.

7. $\mathrm{O}^{\prime}$ Callaghan DS, Humbert M. A Critical Analysis of Survival in Pulmonary Arterial Hypertension. Eur Respir Rev. 2012;21(125):218-22. doi: 10.1183/09059180.00003512.

8. Simonneau G, Montani D, Celermajer DS, Denton CP, Gatzoulis MA, Krowka $M$, et al. Haemodynamic Definitions and Updated Clinical Classification of Pulmonary Hypertension. Eur Respir J. 2019;53(1):1801913. doi: 10.1183/13993003.01913-2018.

9. Galiè N, Humbert M, Vachiery JL, Gibbs S, Lang I, Torbicki A, et al. 2015 ESC/ ERS Guidelines for the Diagnosis and Treatment of Pulmonary Hypertension: The Joint Task Force for the Diagnosis and Treatment of Pulmonary
Fernandes CJ, Calderaro D, Assad APL, Salibe-Filho W, KatoMorinaga LT, Hoette S, Piloto B, Castro MA, Lisboa RP, Silva TAF, Martins MA, Alves-Jr JL, Jardim C, Terra-Filho M, Souza R; Revisão crítica do manuscrito quanto ao conteúdo intelectual importante: Fernandes CJ, Souza R.

\section{Potencial conflito de interesse}

Dr. Caio J. Fernandes - palestras e conselho consultivo da Bayer e Janssen. Dra. Daniela Calderaro - palestras e conselho consultivo da Bayer. Dra. Ana Paula Luppino Assad - conseho consultivo da Janssen. Dr. Jose L. Alves-Jr - palestras e conselho consultivo da Janssen. Dr. Carlos Jardim - palestras e conselho consultivo da Janssen e palestras pela Bayer. Dr. Mario Terra-Filho - palestras e conselho consultivo da Bayer. Dr. Rogerio de Souza - palestras e conselho consultivo da Bayer, GSK e Janssen.

\section{Fontes de financiamento}

O presente estudo não teve fontes de financiamento externas.

\section{Vinculação acadêmica}

Não há vinculação deste estudo a programas de pósgraduação.

Hypertension of the European Society of Cardiology (ESC) and the European Respiratory Society (ERS): Endorsed by: Association for European Paediatric and Congenital Cardiology (AEPC), International Society for Heart and Lung Transplantation (ISHLT). Eur Respir J. 2015;46(4):903-75. doi: 10.1183/13993003.01032-2015.

10. Maron BA, Hess E, Maddox TM, Opotowsky AR, Tedford RJ, Lahm T, et al. Association of Borderline Pulmonary Hypertension with Mortality and Hospitalization in a Large Patient Cohort: Insights from the Veterans Affairs Clinical Assessment, Reporting, and Tracking Program. Circulation. 2016;133(13):1240-8. doi: 10.1161/CIRCULATIONAHA.115.020207.

11. Gavilanes F, Alves JL Jr, Fernandes C, Prada LFL, Jardim CV, Morinaga LT, et al. Left Ventricular Dysfunction in Patients with Suspected Pulmonary Arterial Hypertension. J Bras Pneumol. 2014;40(6):609-16. doi: 10.1590/ S1806-37132014000600004.

12. Maron BA, Brittain EL, Hess E, Waldo SW, Barón AE, Huang S, et al. Pulmonary Vascular Resistance and Clinical Outcomes in Patients with Pulmonary Hypertension: A Retrospective Cohort Study. Lancet Respir Med. 2020;8(9):873-84. doi: 10.1016/S2213-2600(20)30317-9.

13. Simonneau G, Montani D, Celermajer DS, Denton CP, Gatzoulis MA, Krowka $M$, et al. Haemodynamic Definitions and Updated Clinical Classification of Pulmonary Hypertension. Eur Respir J. 2019;53(1):1801913. doi: 10.1183/13993003.01913-2018.

14. Calderaro D, Alves JLJr, Fernandes CJCDS, Souza R. Pulmonary Hypertension in General Cardiology Practice. Arq Bras Cardiol. 2019;113(3):419-28. doi: 10.5935/abc.20190188.

15. Nathan SD, Barbera JA, Gaine SP, Harari S, Martinez FJ, Olschewski H, et al. Pulmonary Hypertension in Chronic Lung Disease and Hypoxia. Eur Respir J. 2019;53(1):1801914. doi: 10.1183/13993003.01914-2018.

16. Fernandes CJCS, Ota-Arakaki JS, Campos FTAF, Corrêa RA, Gazzana MB, Jardim CVP, et al. Brazilian Thoracic Society Recommendations for 
the Diagnosis and Treatment of Chronic Thromboembolic Pulmonary Hypertension. J Bras Pneumol. 2020;46(4): e20200204. doi: 10.36416/1806-3756/e20200204

17. Frost A, Badesch D, Gibbs JSR, Gopalan D, Khanna D, Manes A, et al. Diagnosis of Pulmonary Hypertension. Eur Respir J. 2019;53(1):1801904. doi: 10.1183/13993003.01904-2018.

18. Jaïs X, Olsson KM, Barbera JA, Blanco I, Torbicki A, PeacockA, et al. Pregnancy Outcomes in Pulmonary Arterial Hypertension in the Modern Management Era. Eur Respir J. 2012;40(4):881-5. doi: 10.1183/09031936.00141211.

19. Guillevin L, Armstrong I, Aldrighetti R, Howard LS, Ryftenius H, Fischer A, et al. Understanding the Impact of Pulmonary Arterial Hypertension on Patients' and Carers' Lives. Eur Respir Rev. 2013;22(130):535-42. doi: 10.1183/09059180.00005713.

20. Mereles D, Ehlken N, Kreuscher S, Ghofrani S, Hoeper MM, Halank M, et al. Exercise and Respiratory Training Improve Exercise Capacity and Quality of Life in Patients with Severe Chronic Pulmonary Hypertension. Circulation. 2006;114(14):1482-9. doi: 10.1161/CIRCULATIONAHA.106.618397.

21. Fuster V, Steele PM, Edwards WD, Gersh BJ, McGoon MD, Frye RL. Primary Pulmonary Hypertension: Natural History and the Importance of Thrombosis. Circulation. 1984;70(4):580-7. doi: 10.1161/01.cir.70.4.580.

22. Olsson KM, Delcroix M, Ghofrani HA, Tiede H, Huscher D, Speich R, et al. Anticoagulation and Survival in Pulmonary Arterial Hypertension: Results from the Comparative, Prospective Registry of Newly Initiated Therapies for Pulmonary Hypertension (COMPERA). Circulation. 2014;129(1):57-65. doi: 10.1161/CIRCULATIONAHA.113.004526.

23. Galiè N, Channick RN, Frantz RP, Grünig E, Jing ZC, Moiseeva O, et al. Risk stratification and Medical Therapy of Pulmonary Arterial Hypertension. Eur Respir J. 2019;53(1):1801889. doi: 10.1183/13993003.01889-2018.

24. Langleben D, Orfanos SE, Giovinazzo M, Schlesinger RD, Hirsch AM, Blenkhorn F, et al. Acute Vasodilator Responsiveness and Microvascular Recruitment in Idiopathic Pulmonary Arterial Hypertension. Ann Intern Med. 2015;162(2):154-6. doi: 10.7326/M14-1402.

25. Rich S, Kaufmann E, Levy PS. The Effect of High Doses of Calcium-Channel Blockers on Survival in Primary Pulmonary Hypertension. N Engl J Med. 1992;327(2):76-81. doi: 10.1056/NEJM199207093270203.

26. Sitbon $\mathrm{O}$, Humbert $\mathrm{M}$, Jaïs $\mathrm{X}$, loos $\mathrm{V}$, Hamid AM, Provencher S, et al. Longterm Response to Calcium Channel Blockers in Idiopathic Pulmonary Arterial Hypertension. Circulation. 2005;111(23):3105-11. doi: 10.1161/ CIRCULATIONAHA.104.488486.

27. Stacher E, Graham BB, Hunt JM, Gandjeva A, Groshong SD, McLaughlin WV et al. Modern Age Pathology of Pulmonary Arterial Hypertension. Am J Respir Crit Care Med. 2012;186(3):261-72. doi: 10.1164/rccm.201201-0164OC.

28. Humbert M, Sitbon O, Simonneau G. Treatment of Pulmonary Arterial Hypertension. N Engl J Med. 2004;351(14): 1425-36. doi: 10.1056/ NEJMra040291.

29. Mitchell JA, Ahmetaj-Shala B, Kirkby NS, Wright WR, Mackenzie LS, Reed DM, et al. Role of Prostacyclin in Pulmonary Hypertension. Glob Cardiol Sci Pract. 2014;2014(4):382-93. doi: 10.5339/gcsp.2014.53.

30. Tuder RM, Cool CD, Geraci MW, Wang J, Abman SH, Wright L, et al. Prostacyclin Synthase Expression is Decreased in Lungs from Patients with Severe Pulmonary Hypertension. Am J Respir Crit Care Med. 1999;159(6):1925-32. doi: 10.1164/ajrccm.159.6.9804054.

31. Christman BW, McPherson CD, Newman JH, King GA, Bernard GR, Groves BM, et al. An Imbalance Between the Excretion of Thromboxane and Prostacyclin Metabolites in Pulmonary Hypertension. N Engl J Med. 1992;327(2):70-5. doi: 10.1056/NEJM199207093270202.

32. Barst RJ, Rubin LJ, Long WA, McGoon MD, Rich S, Badesch DB, et al. A Comparison of Continuous Intravenous Epoprostenol (prostacyclin) with Conventional Therapy for Primary Pulmonary Hypertension. N Engl J Med. 1996:334(5):296-301. doi: 10.1056/NEJM199602013340504.

33. Galiè N, Manes A, Branzi A. Prostanoids for Pulmonary Arterial Hypertension. Am J Respir Med. 2003;2(2):123-37. doi: 10.1007/BF03256644.
34. Olschewski H, Simonneau G, Galiè N, Higenbottam T, Naeije R, Rubin LJ et al. Inhaled Iloprost for Severe Pulmonary Hypertension. N Engl J Med. 2002;347(5):322-9. doi: 10.1056/NEJMoa020204.

35. Sitbon O, Channick R, Chin KM, Frey A, Gaine S, Galiè N, et al. Selexipag for the Treatment of Pulmonary Arterial Hypertension. N Engl J Med. 2015;373(26):2522-33. doi: 10.1056/NEJMoa1503184.

36. Kawanabe Y, Nauli SM. Endothelin. Cell Mol Life Sci. 2011;68(2):195-203. doi: 10.1007/s00018-010-0518-0.

37. Chester AH, Yacoub MH. The Role of Endothelin-1 in Pulmonary Arterial Hypertension. Glob Cardiol Sci Pract. 2014;2014(2):62-78. doi: 10.5339/ gcsp.2014.29.

38. Rubin LJ, Badesch DB, Barst RJ, Galie N, Black CM, Keogh A, et al Bosentan Therapy for Pulmonary Arterial Hypertension. N Engl J Med. 2002;346(12):896-903. doi: 10.1056/NEJMoa012212.

39. Pulido T, Adzerikho I, Channick RN, Delcroix M, Galiè N, Ghofrani HA, et al. Macitentan and Morbidity and Mortality in Pulmonary Arterial Hypertension. N Engl J Med. 2013;369(9):809-18. doi: 10.1056/ NEJMoa1213917.

40. Galiè N, Olschewski H, Oudiz RJ, Torres F, Frost A, Ghofrani HA, et al Ambrisentan for the Treatment of Pulmonary Arterial Hypertension: Results of the Ambrisentan in Pulmonary Arterial Hypertension, Randomized, Double-blind, Placebo-controlled, Multicenter, Efficacy (ARIES) Study 1 and 2. Circulation. 2008;117(23):3010-9. doi: 10.1161/ CIRCULATIONAHA.107.742510.

41. Chester AH, Yacoub MH, Moncada S. Nitric Oxide and Pulmonary Arterial Hypertension. Glob Cardiol Sci Pract. 2017;2017(2):14. doi: 10.21542/ gcsp.2017.14.

42. Sim JY. Nitric Oxide and Pulmonary Hypertension. Korean J Anesthesiol. 2010;58(1):4-14. doi: 10.4097/kjae.2010.58.1.4

43. Galiè N, Ghofrani HA, Torbicki A, Barst RJ, Rubin LJ, Badesch D, et al Sildenafil Citrate Therapy for Pulmonary Arterial Hypertension. N Engl J Med. 2005;353(20):2148-57. doi: 10.1056/NEJMoa050010.

44. Boutou AK, Pitsiou G. Treatment of Pulmonary Hypertension with Riociguat: A Review of Current Evidence and Future Perspectives. Expert Opin Pharmacother. 2020;21(10):1145-55. doi: 10.1080/14656566.2020.1727446

45. Ghofrani HA, Galiè N, Grimminger F, Grünig E, Humbert M, Jing ZC, et al Riociguat for the Treatment of Pulmonary Arterial Hypertension. N Engl J Med. 2013;369(4):330-40. doi: 10.1056/NEJMoa1209655.

46. Ghofrani HA, D'Armini AM, Grimminger F, Hoeper MM, Jansa P, Kim NH, et al. Riociguat for the Treatment of Chronic Thromboembolic Pulmonary Hypertension. N Engl J Med. 2013;369(4):319-29. doi: 10.1056/ NEJMoa1209657.

47. Galiè N, Barberà JA, Frost AE, Ghofrani HA, Hoeper MM, McLaughlin VV, et al. Initial Use of Ambrisentan plus Tadalafil in Pulmonary Arterial Hypertension. N Engl J Med. 2015;373(9):834-44. doi: 10.1056/ NEJMoa1413687.

48. Lajoie AC, Lauzière G, Lega JC, Lacasse Y, Martin S, Simard S, et al. Combination Therapy versus Monotherapy for Pulmonary Arterial Hypertension: A Meta-analysis. Lancet Respir Med. 2016;4(4):291-305. doi: 10.1016/S2213-2600(16)00027-8

49. Sitbon O, Jaïs X, Savale L, Cottin V, Bergot E, Macari EA, et al. Upfront Triple Combination Therapy in Pulmonary Arterial Hypertension: A Pilot Study. Eur Respir J. 2014;43(6):1691-7. doi: 10.1183/09031936.00116313.

50. Chin KM, Sitbon O, Doelberg M, Gibbs JSSR, Hoeper MM, Martin N, et al. Efficacy and Safety of Initial Triple Oral Versus Initial Double Oral Combination Therapy in Patients With Newly Diagnosed Pulmonary Arterial Hypertension (PAH): Results of The Randomized Controlled Triton Study. Am J Respir Crit Care Med. 2020; 201:A2928. doi: 10.1164/ajrccmconference.2020.201.1 MeetingAbstracts.A2928. 
51. Coghlan JG, Channick R, Chin K, Di Scala L, Galiè N, Ghofrani HA, et al. Targeting the Prostacyclin Pathway with Selexipag in Patients with Pulmonary Arterial Hypertension Receiving Double Combination Therapy: Insights from the Randomized Controlled GRIPHON Study. Am J Cardiovasc Drugs. 2018;18(1):37-47. doi: 10.1007/s40256-017-0262-z.

52. Fernandes CJCS, Humbert M, Souza R. Challenging the Concept of Adding More Drugs in Pulmonary Arterial Hypertension. Eur Respir J. 2017;50(3):1701527. doi: 10.1183/13993003.01527-2017.

53. Boucly A, Weatherald J, Savale L, Jaïs X, Cottin V, Prevot G, et al. Risk Assessment, Prognosis and Guideline Implementation in Pulmonary Arterial Hypertension. Eur Respir J. 2017;50(2):1700889. doi: 10.1183/13993003.00889-2017.

54. Hoeper MM, Kramer T, Pan Z, Eichstaedt CA, Spiesshoefer J, Benjamin N, et al. Mortality in Pulmonary Arterial Hypertension: Prediction by the 2015 European Pulmonary Hypertension Guidelines Risk Stratification Model. Eur Respir J. 2017; 50(2):1700740. doi: 10.1183/13993003.00740-2017.

55. Kylhammar D, Kjellström B, Hjalmarsson C, Jansson K, Nisell M, Söderberg S, et al. A Comprehensive Risk Stratification at Early Follow-up Determines Prognosis in Pulmonary Arterial Hypertension. Eur Heart J. 2018;39(47):4175-81. doi: 10.1093/eurheartj/ehx257.

56. Benza RL, Gomberg-Maitland M, Elliott CG, Farber HW, Foreman AJ, Frost AE, et al. Predicting Survival in Patients With Pulmonary Arterial Hypertension: The REVEAL Risk Score Calculator 2.0 and Comparison with ESC/ERS-Based Risk Assessment Strategies. Chest. 2019;156(2):323-37. doi: 10.1016/j.chest.2019.02.004.

57. Alves JL Jr, Souza R. Prognostic Relevance of Appropriate Renal Function Evaluation in Pulmonary Arterial Hypertension. J Heart Lung Transplant. 2018;37(6):692-3. doi: 10.1016/j.healun.2018.02.005.

58. Aithala R, Alex AG, Danda D. Pulmonary Hypertension in Connective Tissue Diseases: An Update. Int J Rheum Dis. 2017;20(1):5-24. doi: 10.1111/1756-185X.13001.

59. Alves JL Jr, Gavilanes F, Jardim C, Fernandes CJCDS, Morinaga LTK, Dias B, et al. Pulmonary arterial hypertension in the southern hemisphere: results from a registry of incident Brazilian cases. Chest. 2015;147(2):495-501. doi: 10.1378/chest.14-1036.

60. Badesch DB, Raskob GE, Elliott CG, Krichman AM, Farber HW, Frost AE, etal. Pulmonary Arterial Hypertension: Baseline Characteristics from the REVEAL Registry. Chest. 2010;137(2):376-87. doi: 10.1378/chest.09-1140.

61. Assad APL, Oleas FG, Alves JL Jr, Fernandes CJCS, Sampaio-Barros PD, Souza R. Survival of Connective Tissue Disease Associated Pulmonary Arterial Hypertension. Clin Exp Rheumatol. 2018;36(4):186.

62. Hao YJ, Jiang X, Zhou W, Wang Y, Gao L, Wang Y, et al. Connective Tissue Disease-Associated Pulmonary Arterial Hypertension in Chinese Patients. Eur Respir J. 2014;44(4):963-72. doi: 10.1183/09031936.00182813.

63. Avouac J, Airò P, Meune C, Beretta L, Dieude P, Caramaschi P, et al. Prevalence of Pulmonary Hypertension in Systemic Sclerosis in European Caucasians and Metaanalysis of 5 Studies. J Rheumatol. 2010;37(11):22908. doi: 10.3899/jrheum. 100245.

64. Ruiz-Irastorza G, Garmendia M, Villar I, Egurbide MV, Aguirre C. Pulmonary Hypertension in Systemic Lupus Erythematosus: Prevalence, Predictors and Diagnostic Strategy. Autoimmun Rev. 2013;12(3):410-5. doi: 10.1016/j. autrev.2012.07.010.

65. Kim JS, Kim D, Joo YB, Won S, Lee J, Shin J, et al. Factors Associated With Development and Mortality of Pulmonary Hypertension in Systemic Lupus Erythematosus Patients. Lupus. 2018;27(11):1769-77. doi: $10.1177 / 0961203318788163$.

66. Coghlan JG, Denton CP, Grünig E, Bonderman D, Distler O, Khanna D, et al. Evidence-Based Detection of Pulmonary Arterial Hypertension in Systemic Sclerosis: The DETECT study. Ann Rheum Dis. 2014;73(7):1340-9. doi: 10.1136/annrheumdis-2013-203301.

67. Denton CP, Wells AU, Coghlan JG. Major Lung Complications of Systemic Sclerosis. Nat Rev Rheumatol. 2018;14(9):511-27. doi: 10.1038/s41584018-0062-0.
68. Launay D, Sobanski V, Hachulla E, Humbert M. Pulmonary Hypertension in Systemic Sclerosis: Different Phenotypes. Eur Respir Rev. 2017;26(145):170056. doi: 10.1183/16000617.0056-2017.

69. Desai CS, Lee DC, Shah SJ. Systemic Sclerosis and the Heart: Current Diagnosis and Management. Curr Opin Rheumatol. 2011;23(6):545-54. doi: 10.1097/BOR.0b013e32834b8975.

70. Kiely DG, Lawrie A, Humbert M. Screening Strategies for Pulmonary Arterial Hypertension. Eur Heart J Suppl. 2019;21(Suppl K):9-20. doi: 10.1093/ eurheartj/suz204.

71. Hao Y, Thakkar V, Stevens W, Morrisroe K, Prior D, Rabusa C, et al. A Comparison of the Predictive Accuracy of Three Screening Models for Pulmonary Arterial Hypertension in Systemic Sclerosis. Arthritis Res Ther. 2015;17(1):7. doi: 10.1186/s13075-015-0517-5.

72. Jais X, Launay D, Yaici A, Le Pavec J, Tchérakian C, Sitbon O, et al. Immunosuppressive Therapy in Lupus- and Mixed Connective Tissue Disease-Associated Pulmonary Arterial Hypertension: A Retrospective Analysis of Twenty-three Cases. Arthritis Rheum. 2008;58(2):521-31. doi: 10.1002/art.23303.

73. Sanchez O, Sitbon O, Jaïs X, Simonneau G, Humbert M. Immunosuppressive Therapy in Connective Tissue diseases-Associated Pulmonary Arterial Hypertension. Chest. 2006;130(1):182-9. doi: 10.1378/chest.130.1.182.

74. Hachinski V, Oveisgharan S, Romney AK, Shankle WR. Optimizing the Hachinski Ischemic Scale. Arch Neurol. 2012;69(2):169-75. doi: 10.1001/ archneurol.2011.1698.

75. Speich R, Jenni R, Opravil M, Pfab M, Russi EW. Primary Pulmonary Hypertension in HIV Infection. Chest. 1991;100(5):1268-71. doi: 10.1378/ chest.100.5.1268.

76. Petitpretz P, Brenot F, Azarian R, Parent F, Rain B, Herve P, et al. Pulmonary Hypertension in Patients With Human Immunodeficiency Virus Infection. Comparison with Primary Pulmonary Hypertension. Circulation. 1994;89(6):2722-7. doi: 10.1161/01.cir.89.6.2722.

77. Degano B, Guillaume M, Savale L, Montani D, Jaïs X, Yaici A, et al. HIVAssociated Pulmonary Arterial Hypertension: Survival and Prognostic Factors in the Modern Therapeutic Era. AIDS. 2010;24(1):67-75. doi: 10.1097/ QAD.0b013e328331c65e.

78. Jarrett H, Barnett C. HIV-associated Pulmonary Hypertension. Curr Opin HIV AIDS. 2017;12(6):566-71. doi: 10.1097/COH.0000000000000418.

79. Pergola V, Caruso C, Gnarini R, Fazio S, Ferraro S. Efficacy of Sildenafil in HIVRelated Pulmonary Arterial Hypertension. J Cardiovasc Med. 2015;16(Suppl 2):136-7. doi: 10.2459/JCM.0b013e3283388fb3.

80. Muirhead GJ, Wulff MB, Fielding A, Kleinermans D, Buss N. Pharmacokinetic Interactions Between Sildenafil and Saquinavir/Ritonavir. Br J Clin Pharmacol. 2000;50(2):99-107. doi: 10.1046/j.1365-2125.2000.00245.x.

81. Dejesus E, Saleh S, Cheng S, van der Mey D, Becker C, Frey R, et al. Pharmacokinetic Interaction of Riociguat and Antiretroviral Combination Regimens in HIV-1-Infected Adults. Pulm Circ. 2019;9(2):2045894019848644. doi: 10.1177/2045894019848644.

82. Degano B, Yaïci A, Le Pavec J, Savale L, Jaïs X, Camara B, et al. Long-term Effects of Bosentan in Patients with HIV-Associated Pulmonary Arterial Hypertension. Eur Respir J. 2009;33(1):92-8. doi: 10.1183/09031936.00094808.

83. Aguilar RV, Farber HW. Epoprostenol (Prostacyclin) Therapy in HIVAssociated Pulmonary Hypertension. Am J Respir Crit Care Med. 2000;162(5):1846-50. doi: 10.1164/ajrccm.162.5.2004042.

84. Chinello P, Petrosillo N. Pharmacological Treatment of HIV-Associated Pulmonary Hypertension. Expert Rev Clin Pharmacol. 2016;9(5):715-25. doi: 10.1586/17512433.2016.1151785.

85. Kaufmann P, Niglis S, Bruderer S, Segrestaa J, Äänismaa P, Halabi A, et al. Effect of Lopinavir/Ritonavir on the Pharmacokinetics of Selexipag an Oral Prostacyclin Receptor Agonist and its Active Metabolite in Healthy Subjects. Br J Clin Pharmacol. 2015;80(4):670-7. doi: 10.1111/bcp.12650. 
86. Porres-Aguilar M, Zuckerman MJ, Figueroa-Casas JB, Krowka MJ. Portopulmonary Hypertension: State of the Art. Ann Hepatol. 2008;7(4):321-30. doi: 10.1016/S1665-2681(19)31832-0

87. Alves JL Jr, Gavilanes F, Jardim C, Fernandes CJCDS, Morinaga LTK, Dias B, et al. Pulmonary arterial hypertension in the southern hemisphere: results from a registry of incident Brazilian cases. Chest. 2015;147(2):495-501. doi: 10.1378/chest.14-1036. (Citação repetida (vide no 59). Verificar com os autores a possibilidade de incluir outra citação no lugar da 87 ou se será preciso renumerar todas as citações a partir deste ponto.)

88. Ramsay MA, Simpson BR, Nguyen AT, Ramsay KJ, East C, Klintmalm GB. Severe Pulmonary Hypertension in Liver Transplant Candidates. Liver Transpl Surg. 1997;3(5):494-500. doi: 10.1002/lt.500030503.

89. Le Pavec J, Souza R, Herve P, Lebrec D, Savale L, Tcherakian C, et al. Portopulmonary Hypertension: Survival and Prognostic Factors. Am J Respir Crit Care Med. 2008;178(6):637-43. doi: 10.1164/rccm.200804-613OC

90. Elliott CG, Barst RJ, Seeger W, Porres-Aguilar M, Brown LM, Zamanian RT, et al. Worldwide Physician Education and Training in Pulmonary Hypertension: Pulmonary Vascular Disease: The Global Perspective. Chest. 2010;137(Suppl 6):85-94. doi: 10.1378/chest.09-2816.

91. Cosarderelioglu C, Cosar AM, Gurakar M, Pustavoitau A, Russell SD, Dagher NN, et al. Portopulmonary Hypertension and Liver Transplant: Recent Review of the Literature. Exp Clin Transplant. 2016;14(2):113-20. doi: 10.6002/ect.2015.0351

92. Hoeper MM, Seyfarth HJ, Hoeffken G, Wirtz H, Spiekerkoetter E, Pletz MW, et al. Experience with Inhaled Iloprost and Bosentan in Portopulmonary Hypertension. Eur Respir J. 2007;30(6):1096-102. doi: 10.1183/09031936.00032407

93. Raevens S, Fallon MB. PORTICO: First Randomized Controlled Trial of Vasomodulator Therapy in Portopulmonary Hypertension. Hepatology. 2020;71(5):1870-2. doi: 10.1002/hep.31166.

94. Provencher S, Herve P, Jais X, Lebrec D, Humbert M, Simonneau $\mathrm{G}$, et al. Deleterious Effects of Beta-blockers on Exercise Capacity and Hemodynamics in Patients with Portopulmonary Hypertension. Gastroenterology. 2006;130(1):120-6. doi: 10.1053/j.gastro.2005.10.013.

95. Krowka MJ, Swanson KL, Frantz RP, McGoon MD, Wiesner RH. Portopulmonary Hypertension: Results From a 10-year Screening Algorithm. Hepatology. 2006;44(6):1502-10. doi: 10.1002/hep.21431.

96. Gavilanes F, Fernandes CJ, Souza R. Pulmonary Arterial Hypertension in Schistosomiasis. Curr Opin Pulm Med. 2016;22(5):408-14. doi: 10.1097/ MCP.0000000000000300.

97. Fernandes CJC, Piloto B, Castro M, Oleas FG, Alves JL Jr, Prada LFL, et al. Survival of Patients with Schistosomiasis-Associated Pulmonary Arterial Hypertension in the Modern Management Era. Eur Respir J. 2018;51(6):1800307. doi: 10.1183/13993003.00307-2018.

98. Fernandes CJ, Jardim CV, Hovnanian A, Hoette S, Morinaga LK, Souza R. Schistosomiasis and Pulmonary Hypertension. Expert Rev Respir Med. 2011;5(5):675-81. doi: 10.1586/ers.11.58.

99. Fernandes CJCS, Dias BA, Jardim CVP, Hovnanian A, Hoette S, Morinaga LK, et al. The Role of Target Therapies in Schistosomiasis-Associated Pulmonary Arterial Hypertension. Chest. 2012;141(4):923-8. doi: 10.1378/ chest.11-0483.

100. Hoette S, Figueiredo C, Dias B, Alves JL Jr, Gavilanes F, Prada LFL, et al. Pulmonary Artery Enlargement in Schistosomiasis Associated Pulmonary Arterial Hypertension. BMC Pulm Med. 2015;15:118. doi: 10.1186/ s12890-015-0115-y.

101. Fernandes CJC, Jardim C, Souza R. The Global View. Curr Opin Pulm Med. 2019;25(5):391-7. doi: 10.1097/MCP.0000000000000603.

102. Engelfriet PM, Duffels MG, Möller T, Boersma E, Tijssen JG, Thaulow E, et al. Pulmonary Arterial Hypertension in Adults Born with a Heart Septal Defect: The Euro Heart Survey on Adult Congenital Heart Disease. Heart. 2007:93(6):682-7. doi: 10.1136/hrt.2006.098848.
103. Beghetti M, Galiè N. Eisenmenger Syndrome a Clinical Perspective in a New Therapeutic Era of Pulmonary Arterial Hypertension. J Am Coll Cardiol. 2009;53(9):733-40. doi: 10.1016/j.jacc.2008.11.025.

104. Broberg CS, Ujita M, Prasad S, Li W, Rubens M, Bax BE, et al. Pulmonary Arterial Thrombosis in Eisenmenger Syndrome is Associated with Biventricular Dysfunction and Decreased Pulmonary flow Velocity. J Am Coll Cardiol. 2007;50(7):634-42. doi: 10.1016/j.jacc.2007.04.056.

105. Sandoval J, Aguirre JS, Pulido T, Martinez-Guerra ML, Santos E, Alvarado $\mathrm{P}$, et al. Nocturnal Oxygen Therapy in Patients with the Eisenmenger Syndrome. Am J Respir Crit Care Med. 2001;164(9):1682-7. doi: 10.1164/ ajrccm.164.9.2106076.

106. Galiè N, Beghetti M, Gatzoulis MA, Granton J, Berger RM, Lauer A, et al Bosentan Therapy in Patients with Eisenmenger Syndrome: A Multicenter, Double-blind, Randomized, Placebo-controlled Study. Circulation. 2006;114(1):48-54. doi: 10.1161/CIRCULATIONAHA.106.630715.

107. Rosenzweig EB, Abman SH, Adatia I, Beghetti M, Bonnet D, Haworth $S$, et al. Paediatric Pulmonary Arterial hypertension: Updates on Definition, Classification, Diagnostics and Management. Eur Respir J. 2019;53(1):1801916. doi: 10.1183/13993003.01916-2018.

108. Olsson KM, Jais X. Birth Control and Pregnancy Management in Pulmonary Hypertension. Semin Respir Crit Care Med. 2013;34(5):681-8. doi: 10.1055/s-0033-1355438.

109. Hemnes AR, Kiely DG, Cockrill BA, Safdar Z, Wilson VJ, Al Hazmi M, et al. Statement on Pregnancy in Pulmonary Hypertension from the Pulmonary Vascular Research Institute. Pulm Circ. 2015;5(3):435-65. doi: $10.1086 / 682230$

110. Thorne S, Nelson-Piercy C, MacGregor A, Gibbs S, Crowhurst J, Panay N, et al. Pregnancy and Contraception in Heart Disease and Pulmonary Arterial Hypertension. J Fam Plann Reprod Health Care. 2006;32(2):75-81. doi: 10.1783/147118906776276486.

111. Olsson KM, Channick R. Pregnancy in pulmonary arterial hypertension. Eur Respir Rev. 2016;25(142): 431-7. doi: 10.1183/16000617.0079-2016

112. Sliwa K, van Hagen IM, Budts W, Swan L, Sinagra G, Caruana M, et al Pulmonary Hypertension and Pregnancy Outcomes: Data From the Registry of Pregnancy and Cardiac Disease (ROPAC) of the European Society of Cardiology. Eur J Heart Fail. 2016;18(9):1119-28. doi: 10.1002/ejhf.594.

113. Duarte AG, Thomas S, Safdar Z, Torres F, Pacheco LD, Feldman J, et al. Management of Pulmonary Arterial Hypertension During Pregnancy: A Retrospective, Multicenter Experience. Chest. 2013;143(5):1330-1336. doi: 10.1378/chest.12-0528.

114. Thomas E, Yang J, Xu J, Lima FV, Stergiopoulos K. Pulmonary Hypertension and Pregnancy Outcomes: Insights From the National Inpatient Sample. Am Heart Assoc. 2017;6(10):e006144. doi: 10.1161/JAHA.117.006144.

115. Terek D, Kayikcioglu M, Kultursay H, Ergenoglu M, Yalaz M, Musayev O, et al. Pulmonary Arterial Hypertension and Pregnancy. J Res Med Sci. 2013;18(1):73-6.

116. Madden BP. Pulmonary hypertension and pregnancy. Int J Obstet Anesth. 2009;18(2):156-64. doi: 10.1016/j.ijoa.2008.10.006.

117. Galiè N, Saia F, Palazzini M, Manes A, Russo V, Reggiani MLB, et al. Left Main Coronary Artery Compression in Patients With Pulmonary Arterial Hypertension and Angina. J Am Coll Cardiol. 2017;69(23):2808-2817. doi: 10.1016/j.jacc.2017.03.597.

118. Kajita LJ, Martinez EE, Ambrose JA, Lemos PA, Esteves A, Gama MN, et al. Extrinsic Compression of the Left Main Coronary Artery by a Dilated Pulmonary Artery: Clinical, Angiographic, and Hemodynamic Determinants. Catheter Cardiovasc Interv. 2001;52(1):49-54. doi: 10.1002/1522-726x(200101)52:1<49::aid-ccd1012>3.0.co;2-0.

119. Fernandes Cl, Steigner ML, Piazza G, Goldhaber SZ. Collaborative Cardiology and Pulmonary Management of Pulmonary Hypertension. Chest. 2019;156(2):200-202. doi: 10.1016/j.chest.2019.04.099. 


\section{Artigo de Revisão}

120. Saia F, Dall'Ara G, Marzocchi A, Dardi F, Palazzini M, Manes A, et al. Left Main Coronary Artery Extrinsic Compression in Patients With Pulmonary Arterial Hypertension: Technical Insights and Long-Term Clinical Outcomes After Stenting. JACC Cardiovasc Interv. 2019;12(3):319-21. doi: 10.1016/j. jcin.2018.08.002.

121. Tongers J, Schwerdtfeger B, Klein G, Kempf T, Schaefer A, Knapp JM, et al. Incidence and Clinical Relevance of Supraventricular Tachyarrhythmias in Pulmonary Hypertension. Am HeartJ. 2007;153(1):127-32. doi: 10.1016/j. ahj.2006.09.008.
122. Drakopoulou M, Nashat H, Kempny A, Alonso-Gonzalez R, Swan L, Wort SJ, et al. Arrhythmias in Adult Patients With Congenital Heart Disease and Pulmonary Arterial Hypertension. Heart. 2018;104(23):1963-69. doi: 10.1136/heartjnl-2017-312881. 